\title{
Dynamical interplay of disc thickness and interstellar gas: Implication for the longevity of spiral density waves
}

\author{
Soumavo Ghosh ${ }^{1,2} \odot$ and Chanda J. $\operatorname{Jog}^{3}$

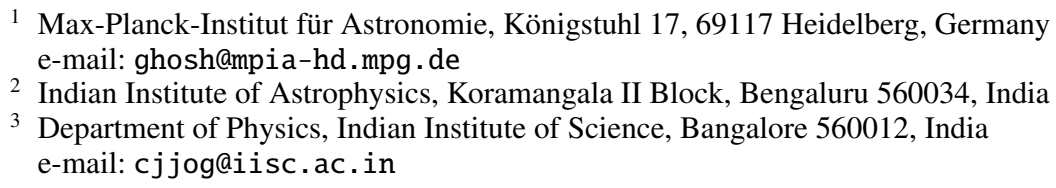

Received 8 September 2021 / Accepted 19 November 2021

\begin{abstract}
A typical galactic disc has a finite thickness. In addition to stars, it also contains a finite amount of interstellar gas. Here, we investigate the physical impact of the finite thickness of a galactic disc on the disc stability against the non-axisymmetric perturbations and on the longevity of the spiral density waves, with and without the presence of gas. The longevity is quantified via the group velocity of density wavepackets. The galactic disc is first modelled as a collisionless stellar disc with finite height and then more realistically as a gravitationally coupled stars plus gas system (with a different thickness for stars and gas). For each case, we derive the appropriate dispersion relation in the Wentzel-Kramers-Brillouin approximation and study the dynamical effect of the disc thickness on the lifetime of spiral density waves via a parametric approach. We find the generic trend that the effective reduction in disc self-gravity due to disc thickness makes it more stable against the non-axisymmetric perturbations and shortens the life-span of the spiral density waves. Furthermore, interstellar gas and disc thickness are shown to have a mutually opposite dynamical effect on the disc stability as well as on the longevity of the spiral density waves. While the gas supports the non-axisymmetric features for a longer time, the disc thickness has an opposite, quenching effect. Consequently, the net change is set by the relative dominance of the opposite effects of the interstellar gas and the disc thickness.
\end{abstract}

Key words. galaxies: evolution - galaxies: kinematics and dynamics - galaxies: spiral - galaxies: structure - instabilities hydrodynamics

\section{Introduction}

Spiral features are one of the most common non-axisymmetric structures (apart from a bar) in disc galaxies in the local Universe (e.g. Elmegreen et al. 2011; Yu et al. 2018; Savchenko et al. 2020). The occurrence of spiral structure in high-redshift (up to $z \sim 1.8$ ) disc galaxies is also known observationally (e.g. Elmegreen \& Elmegreen 2014; Willett et al. 2017; Hodge et al. 2019). The simultaneous occurrence of spiral structure with an $m=2$ bar or an $m=1$ lopsidedness in disc galaxies is common (e.g., see Rix \& Zaritsky 1995; Bournaud et al. 2005; Buta et al. 2010; Zaritsky et al. 2013; Kruk et al. 2018; Ghosh et al. 2021). The Milky Way is a barred spiral galaxy (Weinberg 1992; Gerhard 2002) as well. In the past, a plethora of physical mechanisms ranging from bar-induced spirals (e.g., Salo et al. 2010), tidal encounters (e.g., Toomre \& Toomre 1972; Dobbs et al. 2010), and swing amplification of noise (Goldreich \& Lynden-Bell 1965; Julian \& Toomre 1966; Toomre 1981) due to the disc response to giant molecular clouds (D'Onghia et al. 2013), due to interactions with other spirals (Masset \& Tagger 1997), to recurrent groove modes (Sellwood \& Lin 1989; Sellwood 2012; Sellwood \& Carlberg 2019), and to manifold-driven spirals (Athanassoula 2012) have been proposed for exciting spirals in disc galaxies.

Regardless of the physical mechanism(s) triggering the spiral instability in the disc, it is vital to address the nature and the longevity of spirals. Previous studies (Goldreich \& Lynden-Bell 1965; Julian \& Toomre 1966; Toomre 1981) proposed the spiral arms as transient material arms arising through the joint effects of the epicyclic motion of stars, and shear from differential rotation, and the disc self-gravity. On the other hand, another set of studies by Lin \& Shu $(1964,1966)$ envisaged the spirals as quasi-stationary density waves that rotate with respect to the disc with a well-defined pattern speed (for a detailed recent review, see Shu 2016). Implicit to the formalism of swing amplification, the material arms do not last for a long time (beyond a few dynamical timescales or a few $\times 10^{8} \mathrm{yr}$ ) and are wound up by the disc differential rotation, whereas by definition, the spiral density waves are assumed to be stationary or they last forever. However, Toomre (1969) showed that due to the radial group transport, a wavepacket made of such density waves is eventually destroyed within a timescale of $\sim 1 \mathrm{Gyr}$, thereby posing a challenge to the stationary picture of the density wave theory. Spirals, generated in $N$-body simulations, are almost always found to be a transient phenomenon (e.g. see Sellwood 2011; Grand et al. 2012; Baba et al. 2013, for a review the reader is referred to Dobbs \& Baba 2014). However, the high-resolution $N$-body simulation by D'Onghia et al. (2013) showed that the spirals can be sustained (at least in the statistical sense) for much longer timescales as a result of the non-linear disc response to the perturbation caused by giant molecular cloud-like mass concentration. Another recent work by Saha \& Elmegreen (2016) showed that bulges play a pivotal role in the sustenance of 
spirals in $N$-body simulations, and intermediate-sized bulges can help the spiral density wave to last longer ( $\sim 5 \mathrm{Gyr})$ by providing a high Toomre $Q$-barrier in the inner region. Interstellar gas also is shown to play a pivotal role in the longevity of spiral density waves in disc galaxies. Earlier work by Sellwood \& Carlberg (1984) demonstrated the role of a dissipative component in cooling the stellar disc and facilitating the generation of fresh spiral waves. Jog (1992) included gas along with stars in the framework of the swing amplification mechanism for a more realistic treatment, which yielded broad stellar arms as observed. Furthermore, Ghosh \& Jog $(2015,2016)$ showed that the interstellar gas helps spiral density waves to survive for a longer time (several billion years).

Most of the past analytical studies of spiral structure in disc galaxies treated the galactic disc as being infinitesimally thin for simplicity (but see Goldreich \& Lynden-Bell 1965). This assumption is valid when the height of the disc is small as compared to the wavelength of the perturbation (see discussions in Toomre 1964; Binney \& Tremaine 2008). However, in reality, a galactic disc has a finite height. López-Corredoira \& Molgó (2014) showed that the Galactic disc flares substantially between Galactocentric radii $8 \mathrm{kpc}$ to $25 \mathrm{kpc}$ (also see Li et al. 2019), and the thin-disc component has a scale-height of $\sim 300 \mathrm{pc}$ in the solar neighbourhood (e.g. see Jurić et al. 2008). This flaring of the galactic disc (i.e. increment of the scale height) is a generic phenomenon (e.g. see de Grijs \& Peletier 1997; Narayan \& Jog 2002a; Sarkar \& Jog 2019; García de la Cruz et al. 2021). In addition, the existence of a thick-disc component is now well established observationally in external galaxies as well as in the Milky Way (e.g. see Tsikoudi 1979; Burstein 1979; Yoachim \& Dalcanton 2006; Comerón et al. 2011a,b, 2018). Spirals play a pivotal dynamical role in disc dynamics by transporting angular momentum (Lynden-Bell \& Kalnajs 1972), by causing radial migration of stars without heating (Sellwood \& Binney 2002; Roškar et al. 2008; Schönrich \& Binney 2009), and by exciting vertical breathing motions (Debattista 2014; Faure et al. 2014; Ghosh et al. 2022). Thus, a more realistic study of the spiral structure in disc galaxies should take the finite thickness of the disc into account.

Previous theoretical studies have shown that the introduction of a finite thickness of a galactic disc results in a net reduction in the radial force in the mid-plane (e.g. see Toomre 1964; Jog \& Solomon 1984; Jog 2014). This, in turn, enables the galactic disc to easily become more stable against the axisymmetric perturbations (Toomre 1964; Jog \& Solomon 1984). Julian \& Toomre (1966) demonstrated that the finite thickness of the stellar disc decreases the amplitude of the density transforms in a local patch of the disc. Furthermore, Ghosh \& Jog (2018) showed the generic trend of a suppressed growth of the swing-amplified spirals when a finite thickness for the disc is introduced. However, little is known about the plausible role of the disc finite thickness on the radial group transport and on the longevity of spiral density wave.

In this paper, we investigate the physical effect of the finite thickness of a galactic disc on the persistence of spiral density wave in absence and in presence of the interstellar gas. To achieve this, we first derive the appropriate dispersion relation in the Wentzel-Kramers-Brillouin (WKB) limit for a collisionless stellar disc with a finite thickness and for a gravitationally coupled two-component (stars plus gas) system with different thickness for the stellar and gas discs. Then, we systematically vary different input parameters, namely, the Toomre $Q$ parameter, the thickness of the disc, and the gas fraction, to determine the dependence of the longevity of the spiral density wave, if any, on the finite thickness of the disc. The rest of the paper is organised as follows: Sect. 2 provides the derivation of the relevant dispersion relations in the WKB limit, and Sect. 3 presents the dimensionless form of the corresponding dispersion relations. Section 4 gives our results, which cover the effect of finite thickness on the persistence of the spiral density waves, with and without the interstellar gas. Sections 5 and 6 contain discussion and the main findings of this work, respectively.

\section{WKB dispersion relation for a galactic disc with finite thickness}

Here, we first derive the dispersion relation for a one-fluid disc, and then extend it to a collisionless stellar disc with finite height (Sects. 2.1 and 2.2). Finally, we consider a more realistic gravitationally coupled two-component (stars plus gas) system where the stellar and the gaseous discs can have different scale heights (Sect. 2.3), as observed in real galaxies. The underlying disc is taken to be axisymmetric, and the spiral structure is treated as only a small perturbation on the steady-state axisymmetric disc, so that the linear perturbation approach is valid (for details, see Binney \& Tremaine 2008). A cylindrical coordinate system $(R, \phi, z)$ is used throughout the formulation.

\subsection{One-component fluid disc}

Following the treatment given in Binney \& Tremaine (2008), we started with an infinitesimally thin fluid disc, and then we modified the formulation by introducing the effect due to the finite thickness of the fluid disc. The fluid disc is characterised by the disc surface density $\Sigma_{\mathrm{d}}$ and the sound speed $c$. We assumed that the pressure acts only in the disc plane. For a system like this, the Euler equations of motion in the cylindrical coordinates become

$\frac{\partial v_{R}}{\partial t}+v_{R} \frac{\partial v_{R}}{\partial R}+\frac{v_{\phi}}{R} \frac{\partial v_{R}}{\partial \phi}-\frac{v_{\phi}^{2}}{R}=-\left(\frac{\partial(\Phi+\mathcal{H})}{\partial R}\right)_{z=0}$,

and,

$\frac{\partial v_{\phi}}{\partial t}+v_{R} \frac{\partial v_{\phi}}{\partial R}+\frac{v_{\phi}}{R} \frac{\partial v_{\phi}}{\partial \phi}+\frac{v_{R} v_{\phi}}{R}=-\frac{1}{R}\left(\frac{\partial(\Phi+\mathcal{H})}{\partial \phi}\right)_{z=0}$.

Here, $\mathcal{H}$ is the specific enthalpy of a polytropic fluid with an equation of state $p=K \Sigma_{\mathrm{d}}^{\gamma}$ where $\gamma=(n+1) / n, n$ being the polytropic index, and $K$ being a proportionality constant, and the form of $\mathcal{H}$ is given by (Binney \& Tremaine 2008)

$\mathcal{H}=\frac{\gamma}{\gamma-1} K \Sigma_{\mathrm{d}}^{\gamma-1}$

Assuming the spiral density wave to be a small perturbation, we write $v_{R}=v_{R_{1}} ; v_{\phi}=v_{\phi_{0}}+v_{\phi_{1}}$, where $v_{R_{1}}$ and $v_{\phi_{1}}$ are small perturbations. Further assuming the random motion to be small compared to the rotation, the Euler equation shows that the unperturbed motion gives rise to

$v_{\phi_{0}}=\sqrt{R \frac{\mathrm{d} \Phi_{0}}{\mathrm{~d} R}}=R \Omega(R)$,

where $\Phi_{0}$ is the unperturbed potential and $\Omega(R)$ is the circular frequency. The linear perturbed equations of motion become

$\frac{\partial v_{R_{1}}}{\partial t}+\Omega \frac{\partial v_{R_{1}}}{\partial \phi}-2 \Omega(R) v_{\phi_{1}}=-\left(\frac{\partial\left(\Phi_{1}+\mathcal{H}_{1}\right)}{\partial R}\right)_{z=0}$, 
and

$\frac{\partial v_{\phi_{1}}}{\partial t}+\left[\frac{\mathrm{d}(\Omega R)}{\mathrm{d} R}+\Omega\right] v_{R_{1}}+\Omega \frac{\partial v_{\phi_{1}}}{\partial \phi}=-\frac{1}{R}\left(\frac{\partial\left(\Phi_{1}+\mathcal{H}_{1}\right)}{\partial \phi}\right)_{z=0}$,

where $\mathcal{H}_{1}$ is the perturbed specific enthalpy.

\subsubsection{Introduction of finite thickness of the fluid disc}

For simplicity, we assumed that the disc has a constant density that does not vary with $z$, and the disc has a total thickness of $2 h$. For an infinitesimally thin axisymmetric disc and an axisymmetric perturbation, the solution of the Poisson equation is given by (Toomre 1964)

$\Phi_{1}=-(2 \pi G /|k|) \Sigma_{1} \exp (-k|z|)$,

where $k$ is the wavenumber of perturbation and $\Phi_{1}$ and $\Sigma_{1}$ are the perturbation (or imposed) potential and the corresponding surface density, respectively. The perturbation surface density was taken to have the form $\exp [i(k r-\omega t)]$. For such a disc, the radial force at the mid-plane $(z=0)$ due to a vertical layer between $z$ and $z+\mathrm{d} z$ is proportional to $\exp [-k|z| \mathrm{d} z]$. Hence, the net radial force at $z=0$ is obtained by integrating over $z$ to be (see Toomre 1964)

$\left(\left.\frac{\partial \Phi_{1}}{\partial R}\right|_{z=0}\right)_{\text {net }}=-i 2 \pi G \Sigma_{1} \delta$

where $\delta$ is the reduction factor, which denotes the reduction in the radial force at the mid-plane due to the finite height for a constant density disc. This can also be thought of as a reduction in the disc surface density, and its form is given as (Toomre 1964)

$\delta=[1-\exp (-k h)] / k h$.

In an analogous fashion, by integrating the contribution of force due to layers at different $z$, we can show that the azimuthal force in the mid-plane is also reduced by an identical reduction factor.

We point out that the perturbation potential and the surface density in the framework of the WKB approximation follow the same relation (Eq. (7)) as above (see Eqs. (6)-(18) in Binney \& Tremaine 2008). Hence, a similar analysis (as mentioned above) will yield a same reduction factor $\delta$ (Eq. (9)) in the radial as well as the azimuthal force at mid-plane for the WKB approximation as well. Therefore, in case of a fluid disc with a finite thickness, Eqs. (5) and (6) become

$\frac{\partial v_{R_{1}}}{\partial t}+\Omega \frac{\partial v_{R_{1}}}{\partial \phi}-2 \Omega(R) v_{\phi_{1}}=-\left(\frac{\partial \Phi_{1}}{\partial R} \delta+\frac{\partial \mathcal{H}_{1}}{\partial R}\right)_{z=0}$,

and,

$\frac{\partial v_{\phi_{1}}}{\partial t}+\left[\frac{\mathrm{d}(\Omega R)}{\mathrm{d} R}+\Omega\right] v_{R_{1}}+\Omega \frac{\partial v_{\phi_{1}}}{\partial \phi}=-\frac{1}{R}\left(\frac{\partial \Phi_{1}}{\partial \phi} \delta+\frac{\partial \mathcal{H}_{1}}{\partial \phi}\right)_{z=0}$.

We assumed that the trial solutions are of the form

$$
\begin{aligned}
v_{R_{1}} & =\operatorname{Re}\left[v_{R_{a}}(R) e^{i(m \phi+k R-\omega t)}\right] \\
v_{\phi_{1}} & =\operatorname{Re}\left[v_{\phi_{a}}(R) e^{i(m \phi+k R-\omega t)}\right] \\
\Phi_{1} & =\operatorname{Re}\left[\Phi_{a}(R) e^{i(m \phi+k R-\omega t)-k|z|}\right] \\
\Sigma_{\mathrm{d}_{1}} & =\operatorname{Re}\left[\Sigma_{\mathrm{d}_{a}}(R) e^{i(m \phi+k R-\omega t)}\right] \\
\mathcal{H}_{1} & =\operatorname{Re}\left[\mathcal{H}_{a}(R) e^{i(m \phi+k R-\omega t)}\right] .
\end{aligned}
$$

Putting these in Eqs. (10) and (11), and after some algebraic simplification, we obtain

$v_{R_{a}}=\frac{i}{\Delta}\left[(\omega-m \Omega)\left(\delta \frac{\mathrm{d} \Phi_{a}}{\mathrm{~d} R}+\frac{\mathrm{d} \mathcal{H}_{a}}{\mathrm{~d} R}\right)-\frac{2 m \Omega}{R}\left(\Phi_{a} \delta+\mathcal{H}_{a}\right)\right]$,

and

$v_{\phi_{a}}=-\frac{1}{\Delta}\left[2 B\left(\delta \frac{\mathrm{d} \Phi_{a}}{\mathrm{~d} R}+\frac{\mathrm{d} \mathcal{H}_{a}}{\mathrm{~d} R}\right)+\frac{m(\omega-m \Omega)}{R}\left(\Phi_{a} \delta+\mathcal{H}_{a}\right)\right]$,

where $B$ is the Oort constant and $\Delta \equiv \kappa^{2}-(\omega-m \Omega)^{2}, \kappa$ being the epicyclic frequency. Moroever, the perturbed equation of state gives $\mathcal{H}_{a}=c^{2} \frac{\Sigma_{\mathrm{d}_{a}}}{\Sigma_{0}}$, where $\Sigma_{0}$ is the unperturbed disc surface density (for details, see Binney \& Tremaine 2008).

Similarly, the perturbed continuity equation in cylindrical coordinates is

$\frac{\partial \Sigma_{\mathrm{d}_{1}}}{\partial t}+\Omega \frac{\partial \Sigma_{\mathrm{d}_{1}}}{\partial \phi}+\frac{1}{R} \frac{\partial}{\partial R}\left(R v_{R_{1}} \Sigma_{0}\right)+\frac{\Sigma_{0}}{R} \frac{\partial v_{\phi_{1}}}{\partial \phi}=0$,

which, after substituting the trial solution (Eq. (12)), becomes

$-i(\omega-m \Omega) \Sigma_{\mathrm{d}_{a}} e^{i k R}+\frac{1}{R} \frac{\mathrm{d}}{\mathrm{d} R}\left(R v_{R_{a}} e^{i k R} \Sigma_{0}\right)+\frac{i m \Sigma_{0}}{R} v_{\phi_{a}} e^{i k R}=0$.

\subsubsection{Dispersion relation}

We invoke the WKB approximation to derive an analytical dispersion relation. In this limit, Eqs. (13) and (14) reduce to (for details, see Binney \& Tremaine 2008)

$v_{R_{a}}=-\frac{(\omega-m \Omega)}{\Delta} k\left(\Phi_{a} \delta+\mathcal{H}_{a}\right) \quad$ and $\quad v_{\phi_{a}}=-\frac{2 i B}{\Delta} k\left(\Phi_{a} \delta+\mathcal{H}_{a}\right)$.

The continuity equation (Eq. (16)) also reduces to

$-(\omega-m \Omega) \Sigma_{\mathrm{d}_{a}}+k \Sigma_{0} v_{R_{a}}=0$,

as in the WKB approximation, the last term in Eq. (16) involving $v_{\phi_{a}}$ is smaller than the first two terms and can be dropped (Binney \& Tremaine 2008). By substituting the values for $v_{R_{a}}$, $\mathcal{H}_{a}$ as obtained above and $\Phi_{a}=-2 \pi G \Sigma_{a} /|k|$, as obtained in the WKB limit using Eqs. (7) and (12), where $\Phi_{a}$ and $\Sigma_{a}$ are the imposed perturbation potential and the corresponding imposed perturbation surface density, and after some algebraic manipulation, Eq. (18) reduces to

$\Sigma_{\mathrm{d}_{a}}=\frac{2 \pi G|k| \Sigma_{0} \delta}{\kappa^{2}-(\omega-m \Omega)^{2}+c^{2} k^{2}} \Sigma_{a}$,

where $\Sigma_{\mathrm{d}_{a}}$ is the disc response density. For a self-sustained density wave, the quantities $\Sigma_{a}$ and $\Sigma_{\mathrm{d}_{a}}$ should be equal, and hence the dispersion relation becomes (Binney \& Tremaine 2008)

$(\omega-m \Omega)^{2}=\kappa^{2}-2 \pi G|k| \Sigma_{0} \delta+c^{2} k^{2}$,

where $m$ is a positive integer, denoting the $m$-fold rotational symmetry of the perturbation. We note that in the limit of $h \rightarrow 0$, so that $\delta \rightarrow 1$, the dispersion relation (Eq. (20)) is reduced to the corresponding dispersion relation for an infinitesimally thin fluid disc (see Binney \& Tremaine 2008), as expected. In other words, Eq. (20) is a generalisation of the dispersion relation for an infinitesimally thin fluid disc. Here, the additional factor $\delta$ comes from the inclusion of the effect of finite height in the calculation. 


\subsection{One-component stellar disc}

A cold stellar disc is dynamically equivalent to a fluid disc with zero pressure or zero enthalpy, and hence, the perturbation $\bar{v}_{R_{1}}$ in the mean radial velocity of the stars can be obtained from Eqs. (5) and (6) (assuming zero pressure) as (Binney \& Tremaine 2008)

$\bar{v}_{R_{a}}=-\frac{\omega-m \Omega}{\Delta} k \Phi_{a}$.

Here, $\Delta$ is as defined earlier. If we assume the disc to have a finite thickness of $2 h$ and a constant density $\rho$ (as in Sect. 2.1.1), then in an analogous fashion, the radial force in the mid-plane is reduced by a reduction factor $\delta$ (defined in Eq. (9)). Then following the same procedure as done in Sect. 2.1.1, namely solving the Euler equation (Eq. (10)) with zero enthalpy, the solution for $\bar{v}_{R_{a}}$ is obtained in the WKB limit to be

$\bar{v}_{R_{a}}=-\frac{\omega-m \Omega}{\Delta} k\left(\Phi_{a} \delta\right)$.

As expected, this can be obtained from Eq. (17) by setting the enthalpy to be zero.

If the stellar disc is not sufficiently cold, that is, if the typical epicyclic amplitude is not small enough as compared to the wavelength $(2 \pi / k)$ of the perturbation, then the net response velocity measured at a given location is due to stars with large epicyclic amplitudes and hence would have sampled different values of the spiral potential. This results in a partial cancellation in the mean velocity perturbation response to the imposed potential (for details, see Binney \& Tremaine 2008). Therefore, the resulting expression for the mean velocity perturbation for a thin disc is given by

$\bar{v}_{R_{a}}=-\frac{\omega-m \Omega}{\Delta} k \Phi_{a} \mathcal{F}$,

where $\mathcal{F}$ is the factor by which the response of the disc to a given spiral perturbation is diminished below the value for a cold disc. The form of $\mathcal{F}$ is discussed in Sect. 3.2.

It is straightforward to show that for a stellar disc with finite thickness, where we start with $\bar{v}_{R_{a}}$ as for a finite hight case above (Eq. (22)), and then take account of the reduction in the collisionless disc response when the disc is not cold, hence these two effects manifest, and the expression for the mean velocity perturbation becomes

$\bar{v}_{R_{a}}=-\frac{\omega-m \Omega}{\Delta} k\left(\Phi_{a} \delta\right) \mathcal{F}$.

The perturbed continuity equation in the WKB limit becomes (see e.g., Binney \& Tremaine 2008)

$-(\omega-m \Omega) \Sigma_{\mathrm{d}_{a}}+k \Sigma_{0} \bar{v}_{R_{a}}=0$.

This is identical to Eq. (18) since the continuity equation has the same form for fluid and collisionless cases. By substituting $\bar{v}_{R_{a}}$ from Eq. (24), the above reduces to

$-(\omega-m \Omega) \Sigma_{\mathrm{d}_{a}}+k \Sigma_{0}\left[-\frac{\omega-m \Omega}{\Delta} k\left(\Phi_{a} \delta\right) \mathcal{F}\right]=0$.

Finally, invoking the WKB approximation as before (Sect. 2.1.1), we substitute $\Phi_{a}=-2 \pi G \Sigma_{a} /|k|$ (Sect. 2.2), and obtain

$\Sigma_{\mathrm{d}_{a}}=\frac{2 \pi G \Sigma_{0}|k|}{\Delta} \delta \mathcal{F} \Sigma_{a}$.
Again, for a self-sustained density wave, the disc response surface density $\left(\Sigma_{\mathrm{d}_{a}}\right)$ should be equal to the imposed surface density $\left(\Sigma_{a}\right)$. This in turn yields the dispersion relation for the collisionless disc in the WKB limit as

$(\omega-m \Omega)^{2}=\kappa^{2}-2 \pi G|k| \Sigma_{0} \delta \mathcal{F}$.

Here also we note that in the limit of $h \rightarrow 0$, so that $\delta \rightarrow 1$, the dispersion relation (Eq. (28)) is reduced to the corresponding dispersion relation for an infinitesimally thin collisionless stellar disc (see Binney \& Tremaine 2008), as expected. In other words, Eq. (28) is a generalisation of the dispersion relation for an infinitesimally thin stellar disc. Here, the additional factor $\delta$ comes from the inclusion of the effect of finite height in the calculation.

\subsection{Two-component star-gas system with different finite thickness}

Here, we treat a galactic disc as a gravitationally coupled twocomponent (stars plus gas) system ${ }^{1}$. The stellar disc is taken to be collisionless in nature and is characterised by a surface density $\Sigma_{0 \mathrm{~s}}$, a one-dimensional velocity dispersion, $\sigma_{\mathrm{s}}$, and a total thickness of $2 h_{\mathrm{s}}$, while the gas disc is treated as a fluid and is characterised by the surface density $\Sigma_{0 \mathrm{~g}}$, a one-dimensional velocity dispersion or the sound speed, $c_{\mathrm{g}}$, and a total thickness of $2 h_{\mathrm{g}}$. Since the stars and the gas are gravitationally coupled, their motion will be governed by the joint potential $\left(\Phi_{\text {tot }}\right)$, which is set by both the stellar and the gas discs, that is, $\Phi_{\text {tot }}=\Phi_{\mathrm{s}}+\Phi_{\mathrm{g}}$. The right-hand side (rhs) of Eqs. (1) and (2) will now contain a derivative of the total potential. Thus, the steady-state unperturbed motion will now be given by

$v_{\phi_{0 i}}=\sqrt{R \frac{\mathrm{d} \Phi_{\mathrm{tot}, 0}}{\mathrm{~d} R}}=R \Omega(R)$.

For such a system, the reduction due to the finite height affects the corresponding radial force of each component separately, and each component is affected by the net force due to both components (see e.g. Jog \& Solomon 1984). Hence, the rhs of the perturbed Euler equation (Eq. (10)) becomes $\left(\partial \Phi s_{1} / \partial R\right) \delta_{\mathrm{s}}+\left(\partial \Phi_{\mathrm{g}_{1}} / \partial R\right) \delta_{\mathrm{g}}+\left(\partial \mathcal{H}_{1_{\mathrm{g}}} / \partial R\right)$ for $i=g$ (gas), while for the collisionless stellar disc $(i=s)$, only the first two terms above (without the enthalpy term) are kept, as discussed in Sect. 2.2. The same correction applies to the azimuthal perturbed equation of motion as well. By assuming trial solutions similar to those shown in Eq. (12) and invoking the WKB approximation, the solutions of the Euler equations give the amplitudes of perturbed velocity along the radial and the azimuthal directions for the stellar and the gas disc, respectively, as

$v_{R_{a_{\mathrm{s}}}}=-\frac{(\omega-m \Omega)}{\Delta} k\left(\Phi_{a_{\mathrm{s}}} \delta_{\mathrm{s}} \mathcal{F}+\Phi_{a_{\mathrm{g}}} \delta_{\mathrm{g}}\right)$

$v_{\phi_{a_{\mathrm{s}}}}=-\frac{2 i B}{\Delta} k\left(\Phi_{a_{\mathrm{s}}} \delta_{\mathrm{s}} \mathcal{F}+\Phi_{a_{\mathrm{g}}} \delta_{\mathrm{g}}\right)$

and

$v_{R_{\mathrm{g}}}=-\frac{(\omega-m \Omega)}{\Delta} k\left(\Phi_{a_{\mathrm{s}}} \delta_{\mathrm{s}}+\Phi_{a_{\mathrm{g}}} \delta_{\mathrm{g}}+\mathcal{H}_{a_{\mathrm{g}}}\right)$

$v_{\phi_{a \mathrm{~g}}}=-\frac{2 i B}{\Delta} k\left(\Phi_{a_{\mathrm{s}}} \delta_{\mathrm{s}}+\Phi_{a_{\mathrm{g}}} \delta_{\mathrm{g}}+\mathcal{H}_{a_{\mathrm{g}}}\right)$.

1 The main difference between this work and Ghosh \& Jog (2015) is that here we take the finite thickness of the stellar and the gaseous discs into account. 
Substituting the expression for $v_{R_{\mathrm{s}}}$ from Eq. (30) in the perturbed continuity equation (analog of Eq. (25)), we obtain

$\Sigma_{a_{a_{\mathrm{s}}}}\left[\kappa^{2}-(\omega-m \Omega)^{2}\right]=k \Sigma_{0_{\mathrm{s}}}\left[k\left(\Phi_{a_{\mathrm{s}}} \delta_{1} \mathcal{F}+\Phi_{a_{\mathrm{g}}} \delta_{\mathrm{g}}\right)\right]$.

By substituting the expression for the perturbation potentials in terms of the surface densities in the WKB limit, namely $\Phi_{a_{\mathrm{s}}}=$ $-2 \pi G \Sigma_{a_{\mathrm{s}}} /|k|$ and similarly for $\Phi_{a_{\mathrm{g}}}$, and setting $\Sigma_{a_{\mathrm{s}}}$, the imposed or perturbation stellar surface density equal to $\Sigma_{\mathrm{d}_{\mathrm{s}}}$, the stellar response surface density for a self-consistent solution, the above reduces to

$\frac{\Sigma_{\mathrm{d}_{a_{\mathrm{s}}}}}{\Sigma_{a_{\mathrm{g}}}}=\frac{2 \pi G k \Sigma_{0_{\mathrm{s}}} \delta_{\mathrm{g}}}{\left(\kappa^{2}-(\omega-m \Omega)^{2}-2 \pi G|k| \Sigma_{0_{\mathrm{s}}} \delta_{\mathrm{s}} \mathcal{F}\right)}$.

Similarly for the gas disc, the continuity equation (Eq. (18)) combined with the perturbed velocity component in the WKB limit (Eq. (31)) gives

$\Sigma_{a_{a_{\mathrm{g}}}}\left[\kappa^{2}-(\omega-m \Omega)^{2}\right]=k \Sigma_{0_{\mathrm{g}}}\left[k\left(\Phi_{a_{\mathrm{s}}} \delta_{\mathrm{s}}+\Phi_{a_{\mathrm{g}}} \delta_{\mathrm{g}}+\mathcal{H}_{a_{\mathrm{g}}}\right)\right]$.

By substituting the expression for the perturbation potentials in terms of the surface densities in the WKB limit, namely $\Phi_{a_{\mathrm{s}}}=-2 \pi G \Sigma_{a_{\mathrm{s}}} /|k|$ and similarly for $\Phi_{a_{\mathrm{g}}}$, and furthermore, for self-consistency, setting $\Sigma_{a_{\mathrm{g}}}=\Sigma_{\mathrm{d}_{a \mathrm{~g}}}$, the above equation reduces to

$\frac{\Sigma_{a_{\mathrm{s}}}}{\Sigma_{\mathrm{d}_{a_{\mathrm{g}}}}}=\frac{\left(\kappa^{2}-(\omega-m \Omega)^{2}+k^{2} c_{\mathrm{g}}^{2}-2 \pi G|k| \Sigma_{0_{\mathrm{g}}} \delta_{\mathrm{g}}\right)}{2 \pi G k \Sigma_{0_{\mathrm{g}}} \delta_{\mathrm{s}}}$.

Combining Eqs. (33) and (35) and setting the condition of self-consistency ( $\Sigma_{a_{\mathrm{s}}}=\Sigma_{\mathrm{d}_{\mathrm{s}}}$ and similarly for the gas disc), we obtain the dispersion relation for this joint star-gas system as

$\frac{2 \pi G \Sigma_{0 \mathrm{~s}}|k| \delta_{\mathrm{s}} \mathcal{F}\left(\frac{\omega-m \Omega}{\kappa}, \frac{k^{2} \sigma_{\mathrm{s}}^{2}}{\kappa^{2}}\right)}{\kappa^{2}-(\omega-m \Omega)^{2}}+\frac{2 \pi G \Sigma_{0 \mathrm{~g}} \delta_{\mathrm{g}}|k|}{\kappa^{2}-(\omega-m \Omega)^{2}+c_{\mathrm{g}}^{2} k^{2}}=1$.

We verified that for an infinitesimally thin disc (where the reduction factors $\delta_{i} \rightarrow 1(i=s, g)$, see Eq. (9)), the above is reduced to the dispersion relation for a star-gas case obtained in Ghosh \& Jog (2015), as expected.

We next define

$\alpha_{\mathrm{s}}=\kappa^{2}-2 \pi G \Sigma_{0 \mathrm{~s}} \delta_{\mathrm{s}}|k| \mathcal{F}\left(\frac{\omega-m \Omega}{\kappa}, \frac{k^{2} \sigma_{\mathrm{s}}^{2}}{\kappa^{2}}\right)$

$\alpha_{\mathrm{g}}=\kappa^{2}-2 \pi G \Sigma_{0 \mathrm{~g}} \delta_{\mathrm{g}}|k|+k^{2} c_{\mathrm{g}}^{2}$

$\beta_{\mathrm{s}}=2 \pi G \Sigma_{0 \mathrm{~s}} \delta_{\mathrm{s}}|k| \mathcal{F}\left(\frac{\omega-m \Omega}{\kappa}, \frac{k^{2} \sigma_{\mathrm{s}}^{2}}{\kappa^{2}}\right)$

$\beta_{\mathrm{g}}=2 \pi G \Sigma_{0 \mathrm{~g}} \delta_{\mathrm{g}}|k|$.

Upon substitution in Eq. (36) and after some algebraic simplification, we obtain

$(\omega-m \Omega)^{4}-\left(\alpha_{\mathrm{s}}+\alpha_{\mathrm{g}}\right)(\omega-m \Omega)^{2}+\left(\alpha_{\mathrm{s}} \alpha_{\mathrm{g}}-\beta_{\mathrm{s}} \beta_{\mathrm{g}}\right)=0$.

This is a quadratic equation in $(\omega-m \Omega)^{2}$. Solving it, we obtain

$(\omega-m \Omega)^{2}=\frac{1}{2}\left[\left(\alpha_{\mathrm{s}}+\alpha_{\mathrm{g}}\right) \pm\left\{\left(\alpha_{\mathrm{s}}+\alpha_{\mathrm{g}}\right)^{2}-4\left(\alpha_{\mathrm{s}} \alpha_{\mathrm{g}}-\beta_{\mathrm{s}} \beta_{\mathrm{g}}\right)\right\}^{1 / 2}\right]$.

The additive root for $(\omega-m \Omega)^{2}$ always leads to a positive quantity, hence it always indicates oscillatory perturbations under all conditions (same as for axisymmetric case; see Jog \& Solomon
1984). In order to study the stability of the system and its further consequences, we therefore consider only the negative root, which is

$(\omega-m \Omega)^{2}=\frac{1}{2}\left[\left(\alpha_{\mathrm{s}}+\alpha_{\mathrm{g}}\right)-\left\{\left(\alpha_{\mathrm{s}}+\alpha_{\mathrm{g}}\right)^{2}-4\left(\alpha_{\mathrm{s}} \alpha_{\mathrm{g}}-\beta_{\mathrm{s}} \beta_{\mathrm{g}}\right)\right\}^{1 / 2}\right]$.

We mention that the underlying formalism we present here closely follows that presented in Jog \& Solomon (1984). This builds on the treatment for one-component stellar and gas discs of finite height as in Sects. 2.1 and 2.2. By solving the coupled equations, the resulting dispersion relation is given by Eq. (36), which has the solution Eq. (40). Moreover, we caution that the form for the solution of the dispersion relation (Eq. (40)) and the subsidiary variables $\alpha_{\mathrm{s}}, \alpha_{\mathrm{g}}, \beta_{\mathrm{s}}$, and $\beta_{\mathrm{g}}$ (Eq. (37)) (in terms of which Eq. (40) is written) may appear similar to that in Jog \& Solomon (1984, who treated a two-fluid case), and Ghosh \& Jog (2015, who treated a star-gas case in which the effect of stellar dispersion was included in terms of the reduction factor $\mathcal{F}$ ). These variables were defined to have a similar form by construction because all three are two-component formulations and have a similar underlying mathematical symmetry, but with a different treatment for stars and gas, and the current work includes the effect of finite height.

\section{Dimensionless form of the dispersion relations in the WKB limit}

In the literature, the dispersion relations are typically described in terms of some dimensionless quantities for the sake of convenience. Here, we follow the same procedure for the dispersion relations derived in the previous sections.

\subsection{One-component fluid disc with finite thickness}

Dividing the two sides of Eq. (20) by $\kappa^{2}$, and after some algebraic simplification, we obtain

$s^{2}=1-x \delta+\frac{1}{4} x^{2} Q^{2}$,

where $s=(\omega-m \Omega) / \kappa$, and $x\left(=|k| / k_{\text {crit }}\right)$ are the dimensionless frequency and wavenumber of the perturbation, respectively; and $k_{\text {crit }}\left(=\kappa^{2} / 2 \pi G \Sigma_{0}\right)$ is the largest stable wavenumber for a pressureless stellar disc. $Q\left(=\kappa c / \pi G \Sigma_{0}\right)$ is the usual Toomre $Q$ parameter for a fluid disc (Toomre 1964). Also, $|k| h$ can be expressed as $|k| h=\left(|k| / k_{\text {crit }}\right) \times\left(k_{\text {crit }} h\right)=x \beta$. Here, $\beta$ is defined to be equal to $k_{\text {crit }} \times h$. Therefore, the dispersion relation becomes

$s^{2}=1-x \delta+\frac{1}{4} x^{2} Q^{2}$,

where the form of $\delta$ reduces to

$\delta=\frac{1-\exp (-x \beta)}{x \beta}$

The value of $\beta$ is dependent on the chosen values of $k_{\text {crit }}$, that is, for the same thickness of a disc, the values of $\beta$ will be different depending on the values of $k_{\text {crit }}$. We note that in the solar neighbourhood, a circular velocity $\left(v_{\mathrm{c}}\right)$ of $\sim 220 \mathrm{~km} \mathrm{~s}^{-1}$ and $\Sigma \sim$ $45 M_{\odot} \mathrm{pc}^{-2}$ (e.g. see Mera et al. 1998; Narayan \& Jog 2002b) will produce $k_{\text {crit }} \sim 1 \mathrm{kpc}^{-1}$. However, recent studies have reported slightly different values for the circular velocity and 
the solar position (e.g., see Gillessen et al. 2009; Schönrich et al. 2010, 2019; Schönrich 2012; McMillan et al. 2018). The galactocentric distance of the Sun is $8.27 \mathrm{kpc}$ (Schönrich 2012), which is in agreement with the other measurements (within their error bars; Gillessen et al. 2009; McMillan et al. 2018). Furthermore, the circular velocity at the Solar radius is $237.8 \mathrm{~km} \mathrm{~s}^{-1}$ (Schönrich et al. 2010), which in turn gives a circular frequency at the solar radius of $28.8 \mathrm{~km} \mathrm{~s}^{-1} \mathrm{kpc}^{-1}$. Assuming a flat rotation curve, the corresponding epicyclic frequency becomes $40.7 \mathrm{~km} \mathrm{~s}^{-1} \mathrm{kpc}^{-1}$. We use these latest values of $\Omega$ and $\kappa$ and consider the group transport at the solar neighbourhood $(R=$ $8.27 \mathrm{kpc})$ in the subsequent sections, unless stated otherwise. Using these recent values, we estimate $k_{\text {crit }} \sim 1.3 \mathrm{kpc}^{-1}$. Therefore, for the sake of uniformity, we chose $k_{\text {crit }}=1.3 \mathrm{kpc}^{-1}$ for all cases considered here, unless stated otherwise. The resulting behaviour of the reduction factor $\delta$ is shown in Appendix A.

\subsection{One-component stellar disc with finite thickness}

Dividing the two sides of Eq. (28) by $\kappa^{2}$, and after some algebraic simplification, we obtain

$s^{2}=1-x \delta \mathcal{F}(s, \chi)$,

where $\chi=k^{2} \sigma_{\mathrm{s}}^{2} / \kappa^{2}=0.286 Q_{\mathrm{s}}^{2} x^{2}$, and $\delta$ is already given in Eq. (43). The form for $\mathcal{F}$ for a razor-thin disc whose stellar equilibrium state is described by the Schwarzschild distribution function is given by (Binney \& Tremaine 2008)

$\mathcal{F}(s, \chi)=\frac{2}{\chi} \exp (-\chi)\left(1-s^{2}\right) \sum_{n=1}^{\infty} \frac{I_{n}(\chi)}{1-s^{2} / n^{2}}$,

where $I_{n}$ is the modified Bessel function of the first kind.

\subsection{Two-component star-gas system with different finite thickness}

Proceeding as before, by dividing the two sides of Eq. (40) by $\kappa^{2}$, and after some algebraic simplification, we obtain

$s^{2}=\frac{1}{2}\left[\left(\alpha_{\mathrm{s}}^{\prime}+\alpha_{\mathrm{g}}^{\prime}\right)-\left\{\left(\alpha_{\mathrm{s}}^{\prime}+\alpha_{\mathrm{g}}^{\prime}\right)^{2}-4\left(\alpha_{\mathrm{s}}^{\prime} \alpha_{\mathrm{g}}^{\prime}-\beta_{\mathrm{s}}^{\prime} \beta_{\mathrm{g}}^{\prime}\right)\right\}^{1 / 2}\right]$,

where

$\alpha_{\mathrm{s}}^{\prime}=1-(1-\epsilon) x \delta_{\mathrm{s}} \mathcal{F}(s, \xi)$

$\alpha_{\mathrm{g}}^{\prime}=1-\epsilon x \delta_{\mathrm{g}}+\frac{1}{4} Q_{\mathrm{g}}^{2} \epsilon^{2} x^{2}$

$\beta_{\mathrm{s}}^{\prime}=(1-\epsilon) x \delta_{\mathrm{s}} \mathcal{F}(s, \xi)$

$\beta_{\mathrm{g}}^{\prime}=\epsilon x \delta_{\mathrm{g}}$

where $\xi=k^{2} \sigma_{\mathrm{s}}^{2} / \kappa^{2}=0.286 Q_{\mathrm{s}}^{2}(1-\epsilon)^{2} x^{2}$. The three dimensionless parameters $Q_{\mathrm{s}}, Q_{\mathrm{g}}$ and $\epsilon$ are the Toomre $Q$ parameters for stars $Q_{\mathrm{s}}\left(=\kappa \sigma_{\mathrm{s}} /\left(3.36 G \Sigma_{0 \mathrm{~s}}\right)\right)$, and for gas $Q_{\mathrm{g}}=\left(\kappa c_{\mathrm{g}} /\left(\pi G \Sigma_{0 \mathrm{~g}}\right)\right)$ and, $\epsilon=\Sigma_{0 \mathrm{~g}} /\left(\Sigma_{0 \mathrm{~s}}+\Sigma_{0 \mathrm{~g}}\right)$ the gas mass fraction in the disc, respectively. Furthermore, the forms of $\delta_{i}$ are given by

$\delta_{i}=\frac{1-\exp \left(-x \beta_{i}\right)}{x \beta_{i}}$.

where $i=s, g$ for stars and gas, respectively. We note that when the height $h_{i} \rightarrow 0$, and so $\delta_{i} \rightarrow 1$, Eq. (46) is reduced to the dispersion relation for the gravitationally coupled twocomponent (stars plus gas) system, which is infinitesimally thin (see Ghosh \& Jog 2015), as expected.

\section{Results}

Here, we present the results related to the dynamical effect of inclusion of a finite thickness of the disc, with or without the presence of the interstellar gas on the disc stability and the longevity of a spiral density wave. For a bi-symmetric $(m=2)$ spiral density wave, the pattern speed $\Omega_{\mathrm{p}}=\omega / 2$ and $s=$ $(\omega-2 \Omega) / \kappa=2\left(\Omega_{\mathrm{p}}-\Omega\right) / \kappa$. Here, $s=0$ corresponds to the corotation (CR) point and $|s|=1$ gives the Lindblad resonances (Ghosh \& Jog 2016).

To quantify the disc stability, we define a quantity $|s|_{\text {cut-off }}$ as the lowest value of the dimensionless frequency $(s)$, corresponding to an appropriate dispersion relation, for which one is able to obtain a real or stable wave solution at any given $R$ (for details, see e.g. Binney \& Tremaine 2008; Ghosh \& Jog 2016). For $s$ lying in the forbidden region, namely, between the corotation or $s=0$ and $s_{\text {cut-off }}$, the solution is imaginary and the wave is transient at any given radius $R$ (for details, see the discussions in Binney \& Tremaine 2008; Ghosh \& Jog 2016). In other words, the value of $|s|_{\text {cut-off }}$ denotes the edge of the forbidden region. A decrease in the value of $|s|_{\text {cut-off }}$ signifies the decrease in the forbidden zone; this causes the disc to become more prone to being unstable against the perturbations. Conversely, an increase in the value of $|s|_{\text {cut-off }}$ denotes an increase in the forbidden zone, and the disc becomes more stable against the perturbations.

As for the longevity of the spiral density wave, following Toomre (1969), we studied the radial group transport of a wavepacket of such a density wave. To achieve this, we calculated the radial group velocity from the local dispersion relation, appropriate for a particular system considered here. It is known that the information from a disturbance, generated at a certain radius $R$, propagates in the disc with its group velocity $v_{\mathrm{g}}$. When the medium is inhomogeneous, the group velocity, at a given radius $R$, is defined as (e.g. see Whitham 1960; Lighthill 1965)

$v_{\mathrm{g}}(R)=\frac{\partial \omega(k, R)}{\partial k}$.

The value of the group velocity $\left(v_{\mathrm{g}}\right)$ at a given $R$ can be estimated from the slope of the local dispersion relation (when expressed in a dimensionless form) by using the following equation (e.g. see Toomre 1969; Binney \& Tremaine 2008):

$v_{\mathrm{g}}(R)=\operatorname{sgn}(k s)\left(\frac{\kappa}{k_{\text {crit }}}\right) \frac{\mathrm{d} s}{\mathrm{~d} x}$.

Here, $s$ and $x$ are the dimensionless frequency and the wavenumber of the perturbation, respectively, and $\operatorname{sgn}(k s)= \pm 1$ depending on whether $k s>0$ or $k s<0$. Here, the slope is obtained at a point $x$ at which the observed value of $s$ intersects the dispersion relation curve. Thus, the location (or the $x$ value) where the slope is to be calculated is determined by the pattern speed $\left(\Omega_{\mathrm{p}}\right)$ value as well as by the underlying mass distribution (which in turn sets the values of $\Omega$ and $\kappa$ ). For more details, see Sect. 4.1.

A decrease in the group velocity implies that a wavepacket consisting of such density waves would take a longer time to reach the centre of the disc, and it would eventually be absorbed. In other words, a decrease in the group velocity signifies a longer persistence of the spiral density waves in the disc (for further discussions, see, e.g. Toomre 1969; Ghosh \& Jog 2015). Section 4.1 provides the details of the effect of finite thickness on the disc instability and longevity of spiral density wave for the one-component stellar disc, and Sect. 4.2 does the same for the gravitationally coupled two-component (stars plus gas) disc. We mention that the method of investigating the disc stability against 


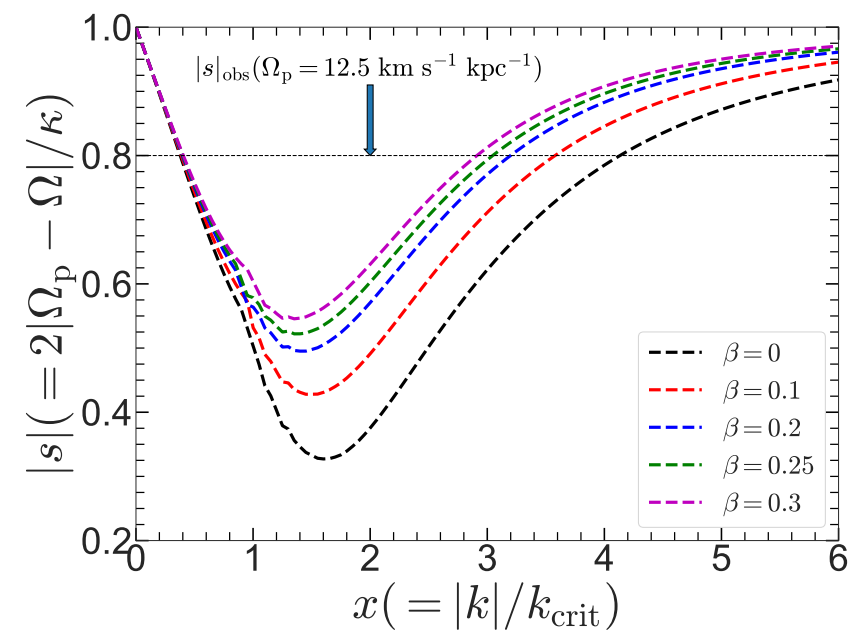

Fig. 1. Dispersion relation for a one-component stellar disc (Eq. (44)) shown for $Q=1.1$ and for $\beta=0.1-0.3$. For comparison, the corresponding dispersion relation for the zero-thickness (or infinitesimally thin) stellar disc is also shown (dashed black line). The horizontal dashed line (in black) denotes the $|s|_{\text {obs }}$ value (calculated at $R=8.27 \mathrm{kpc}$ ) corresponding to an assumed pattern speed of $12.5 \mathrm{~km} \mathrm{~s}^{-1} \mathrm{kpc}^{-1}$.

the $m=2$ spiral density wave is similar to that in Ghosh \& Jog (2016), whereas the treatment for calculating the group velocity is similar to that in Ghosh \& Jog (2015), but here we use the appropriate dispersion relations for a one-component stellar disc, and a two-component disc with finite height, as derived in the previous section.

\subsection{Effect of finite thickness on a one-component stellar disc}

To study the dynamical effect of the disc finite thickness, first we calculated the dispersion relation (Eq. (44)) for Toomre $Q=1.1$ while varying the disc thickness, or equivalently, $\beta$ from 0.1 to 0.3 . Figure 1 shows the corresponding dispersion relations. For comparison, we also show the corresponding dispersion relation for an infinitesimally thin stellar disc with $Q=1.1$. Toomre (1969) assumed an $Q=1$, which denotes the neutral stability of the stellar disc. Here, we instead assumed a slightly higher value of $Q=1.1$ such that the stellar disc is stable against axisymmetric perturbation, but its self-gravity is still important (for details, see Toomre 1964; Jog \& Solomon 1984).

\subsubsection{Disc stability against spiral density waves}

A visual inspection of Fig. 1 reveals that with increasing thickness of the disc, the $|s|_{\text {cut-off }}$ values increase steadily. This implies that the stellar disc becomes increasingly more stable against the non-axisymmetric perturbations. In order to study this more quantitatively, we calculated the $|s|_{\text {cut-off }}$ values from the dispersion relation while systematically varying the Toomre $Q$ values from 1.1 to 2 , and $\beta=0.1,0.3$, and 0.5 . The resulting variation in the $|s|_{\text {cut-off }}$ values for different $\beta$ and Toomre $Q$ parameter values are shown in Fig. 2. For reference, we also show the corresponding $|s|_{\text {cut-off }}$ values for a one-component stellar disc with zero thickness (i.e. $\beta=0$ ). As Fig. 2 clearly shows, for a fixed Toomre $Q$ parameter, the $|s|_{\text {cut-off }}$ value increases monotonically with increasing $\beta$ values. Although this trend remains true for the whole range of Toomre $Q$ values considered here, the effect of the increase in the $|s|_{\text {cut-off }}$ value due to disc thickness is

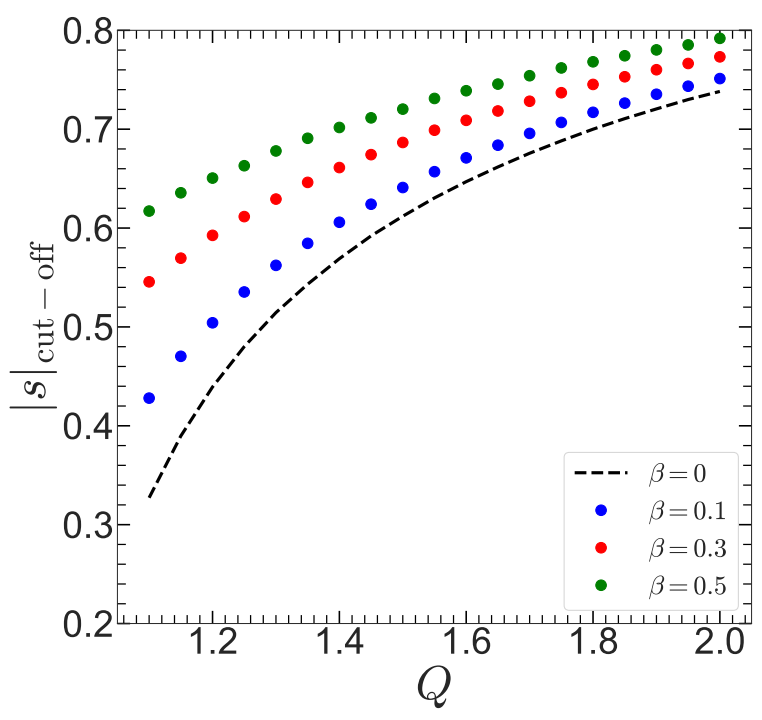

Fig. 2. One-component stellar disc: variation in the $|s|_{\text {cut-off }}$ values as a function of the Toomre $Q$ parameter are shown for different disc thickness $(\beta)$. The dashed black line shows the corresponding $|s|_{\text {cut-off }}$ values for a one-component stellar disc that is infinitesimally-thin. For a fixed Toomre $Q$, the $|s|_{\text {cut-off }}$ values increase monotonically with $\beta$.

more prominent for the lower Toomre $Q$ values considered here. For example, for $Q=1.1$, the $|s|_{\text {cut-off }}$ values increase almost by a factor of 2 when $\beta$ is changed from 0 (zero thickness) to $0.5(\sim 385 \mathrm{pc})$, but the corresponding change is much smaller, by $\sim 7 \%$, for $Q=2$. This trend is not surprising because for lower values of $Q$, the disc self-gravity is more relevant, and consequently, the reduction in self-gravity (due to the finite thickness) will be more pronounced than for the case with a higher Toomre $Q$ value. The physical implications of the increasing $|s|_{\text {cut-off }}$ value with thickness is discussed below.

\subsubsection{Effect of finite thickness on the allowed range of pattern speed values}

As the $|s|_{\text {cut-off }}$ value increases with increasing thickness, this implies that the forbidden region (i.e., the region between the $\mathrm{CR}$ and $\left.|s|_{\text {cut-off }}\right)$ increases as well. In other words, introducing a finite thickness of the disc helps to stabilise it against the nonaxisymmetric perturbations. The increase in the $|s|_{\text {cut-off }}$ value with increasing thickness has far-reaching impact on the pattern speed $\left(\Omega_{p}\right)$ value of a stable, spiral density wave. In a collisionless stellar disc, a spiral density wave exists only in regions where

$\Omega-\kappa / 2 \leq \Omega_{\mathrm{p}} \leq \Omega+\kappa / 2$

is satisfied, and the equality holds only at the Lindblad resonance points (for details, see, e.g. Binney \& Tremaine 2008). Moreover, at a certain radius $R$ and for a given pattern speed $\left(\Omega_{\mathrm{p}}\right)$, the density wave will be stable if the corresponding $|s|\left(=m\left|\Omega_{\mathrm{p}}-\Omega\right| / \kappa\right)$ is greater than the $|s|_{\text {cut-off }}$ value, for instance $\alpha$, of the corresponding dispersion relation at that radius $R$ (for a detailed discussion, see Binney \& Tremaine 2008; Ghosh \& Jog 2016). In other words, to obtain a stable spiral density wave with a pattern speed $\Omega_{\mathrm{p}}$, the following condition needs to be satisfied

$\Omega_{\mathrm{p}} \geq \Omega+\alpha \kappa / 2$ or, $\Omega_{\mathrm{p}} \leq \Omega-\alpha \kappa / 2$

at radius $R$, depending on whether the radius $R$ falls outside the CR or inside the CR. Therefore, by combining Eqs. (51) 
and (52), the allowed range of pattern speed $\left(\Omega_{\mathrm{p}}\right)$ values for a stable spiral density wave is

$\Omega_{\mathrm{p}} \in[\Omega-\kappa / 2, \Omega-\alpha \kappa / 2] \quad$ or, $\Omega_{\mathrm{p}} \in[\Omega+\alpha \kappa / 2, \Omega+\kappa / 2]$, (53)

depending on whether the radius $R$ is inside the corotation or outside the corotation. As the value of $\alpha$ (or $|s|_{\text {cut-off }}$ ) increases with thickness, the allowed range of pattern speed values for a stable spiral density wave become progressively narrower. This is another finding of this work. We note that including gas allows a wider range of allowed pattern speed values (Ghosh \& Jog 2016). Thus, the effects of the gas and the disc thickness have an opposite effect on the range of the pattern speed values, corresponding to a stationary (non-evanescent) spiral density wave. This is further discussed in Sect. 4.2.

\subsubsection{Radial group transport and effect on the longevity of spiral density waves}

Next, we studied whether the radial group transport, and hence the longevity of a spiral density wave, is altered when the finite thickness of the collisionless stellar disc is included. In classical density wave theory, the pattern speed $\left(\Omega_{p}\right)$ of the spiral arms is a free parameter (e.g. see Lin \& Shu 1964, 1966). Observationally, the pattern speed of spiral density waves has been measured only for a few external galaxies (e.g. Fathi et al. 2007, 2009) in addition to the Milky Way. Therefore, driven by purely theoretical interest, we treated the pattern speed $\left(\Omega_{\mathrm{p}}\right)$ as a free parameter. To investigate the effect of disc thickness on the group velocity, we first assumed the pattern speed to be $\Omega_{\mathrm{p}}=12.5 \mathrm{~km} \mathrm{~s}^{-1}$, the same value as used in Toomre (1969). We also chose $Q=1.1$ here, and varied $\beta$ from 0 to 0.3 . Using the definition of the dimensional quantity $s$ as given earlier in this section (i.e. $\left.|s|=2\left(\left|\Omega_{\mathrm{p}}-\Omega\right|\right) / \kappa\right)$ and using $\Omega$ and $\kappa$ values for a particular galaxy at a given radius, and a given value of $\Omega_{\mathrm{p}}$ gives $|s|_{\mathrm{obs}}$, the observed value of $|s|$. This is shown by the horizontal line in Fig. 1. Then, we computed the group velocity $\left(v_{\mathrm{g}}\right)$ at $R=8.27 \mathrm{kpc}$ from the slopes of the corresponding dispersion relations (as shown in Fig. 1) with varying $\beta$ values. The slope is obtained where the line $|s|_{\text {obs }}$ intersects the dispersion relation. The resulting values of the group velocity $\left(v_{\mathrm{g}}\right)$ and the time that one such wavepacket (of density wave) would take to travel a distance of $10 \mathrm{kpc}$ are listed in Table 1 . Before we proceed to interpret the dynamical effect of the thickness on the group velocity, the location at which the slopes are calculated merits a discussion. For an assumed $\Omega_{\mathrm{p}}$ value and the values of $\Omega$ and $\kappa$ (set by the underlying mass distribution), the corresponding $|s|_{\text {obs }}$ would intersect the dispersion relations at two points: one at the long-wavelength branch (lower $x$ value), and another at a short-wavelength branch (higher $x$ value). For a given $\Omega_{\mathrm{p}}$ value, we always calculated the group velocity in the short-wavelength branch regime because the WKB approximation works better there (for details, see Binney \& Tremaine 2008).

Table 1 clearly shows that for $\Omega_{\mathrm{p}}=12.5 \mathrm{~km} \mathrm{~s}^{-1} \mathrm{kpc}^{-1}$ and $Q=1.1$, the value of the group velocity increases steadily with increasing disk thickness $(\beta)$. As the $\beta$ value changes from 0 to 0.3 , the group velocity $\left(v_{\mathrm{g}}\right)$ increases by $\sim 35 \%$. This implies that a wavepacket would take less time to reach the centre of the disc (and eventually become absorbed) for a disc with finite thickness when compared to that of an infinitesimally thin disc. In other words, a spiral density wave would survive for a shorter time for a stellar disc with finite thickness when compared to an infinitesimally thin stellar disc. Next, we chose $\Omega_{\mathrm{p}}=10 \mathrm{~km} \mathrm{~s}^{-1} \mathrm{kpc}^{-1}$ and $15 \mathrm{~km} \mathrm{~s}^{-1} \mathrm{kpc}^{-1}$ and recalculated the variation in the group velocity with changing disc thickness.
Table 1. Group velocity for a one-component stellar disc (with $Q=1.1$ ) for various thickness values, calculated at $R=8.27 \mathrm{kpc}$.

\begin{tabular}{lccccc}
\hline \hline $\begin{array}{l}\Omega_{\mathrm{p}} \\
\left(\mathrm{km} \mathrm{s}^{-1} \mathrm{kpc}^{-1}\right)\end{array}$ & $|s|_{\text {obs }}$ & $\beta$ & $\mathrm{d} s / \mathrm{d} x$ & $\begin{array}{c}v_{\mathrm{g}} \\
\left(\mathrm{km} \mathrm{s}^{-1}\right)\end{array}$ & $\begin{array}{c}\text { Time to travel } \\
10 \mathrm{kpc}(\mathrm{Gyr})\end{array}$ \\
\hline 10 & 0.92 & 0 & 0.032 & 1.3 & 7.5 \\
& & 0.1 & 0.04 & 1.62 & 6.03 \\
& & 0.2 & 0.045 & 1.83 & 5.3 \\
& & 0.25 & 0.047 & 1.91 & 5.1 \\
& & 0.3 & 0.05 & 2.03 & 4.8 \\
\hline 12.5 & 0.8 & 0 & 0.107 & 4.34 & 2.25 \\
& & 0.1 & 0.123 & 5 & 1.95 \\
& & 0.2 & 0.137 & 5.56 & 1.75 \\
& & 0.25 & 0.142 & 5.77 & 1.69 \\
& & 0.3 & 0.143 & 5.81 & 1.68 \\
\hline 15 & 0.68 & 0 & 0.181 & 7.35 & 1.32 \\
& & 0.1 & 0.199 & 8.1 & 1.2 \\
& & 0.2 & 0.203 & 8.25 & 1.18 \\
& & 0.25 & 0.204 & 8.29 & 1.17 \\
& & 0.3 & 0.205 & 8.3 & 1.16 \\
\hline
\end{tabular}

Here also we chose $Q=1.1$. The corresponding results are also given in Table 1. We find that for these pattern speed values, the group velocity increases with the disc thickness, similar to the case of $\Omega_{\mathrm{p}}=12.5 \mathrm{~km} \mathrm{~s}^{-1} \mathrm{kpc}^{-1}$. However, the amount by which the group velocity changes varies with the assumed pattern speed values. To elaborate, the group velocity increases by $\sim 56 \%$ for $\Omega_{\mathrm{p}}=10 \mathrm{~km} \mathrm{~s}^{-1} \mathrm{kpc}^{-1}$, whereas the group velocity increases by $\sim 13 \%$ for $\Omega_{\mathrm{p}}=15 \mathrm{~km} \mathrm{~s}^{-1} \mathrm{kpc}^{-1}$ when the thickness $(\beta)$ is increased from 0 to 0.3 . We also considered a higher Toomre $Q$ value, namely, $Q=1.5$, and studied the variation in the group velocity with disc thickness. For the sake of brevity, the detailed variations in the group velocity values are not shown here. However, we find that for $Q=1.5$ and $\Omega_{\mathrm{p}}=12.5 \mathrm{~km} \mathrm{~s}^{-1} \mathrm{kpc}^{-1}$, the corresponding group velocity $\left(v_{\mathrm{g}}\right)$ increases by $\sim 16 \%$ for an increase in thickness from $\beta=0$ to $\beta=0.3$, as compared to $\sim 35 \%$ for $Q=1.1$ obtained earlier.

We note that the measured group velocity critically depend on the location at which the slope is being measured along the dispersion relation. Nevertheless, an increment in the group velocity value with increasing disc thickness is seen to be a generic phenomenon, as shown here. Lastly, we point out that for the one-component stellar disc case, we could not explore a higher Toomre $Q$ value and/or a higher pattern speed value because for these cases, the measured $|s|_{\text {obs }}$ value is found to be lower that the $|s|_{\text {cut-off }}$ value. Consequently, the $|s|_{\text {obs }}$ does not intersect the corresponding dispersion relation. In other words, for higher Toomre $Q$ values and a higher pattern speed value, for example, $\Omega_{\mathrm{p}}=18 \mathrm{~km} \mathrm{~s}^{-1} \mathrm{kpc}^{-1}$ does not admit a real solution in $k$ (or equivalently, $x$ ), and so we could not calculate the group velocity using Eq. (49). We explore this parameter regime in the next section where the interstellar gas is taken into account.

\subsubsection{Radial variation of the group transport}

So far, we have calculated the group velocity of a typical wavepacket, consisting of a density waves, at a certain radius, $R$, to study the effect of the finite thickness of a stellar disc on the longevity of the spiral density wave. We have so far considered the group transport in the solar neighbourhood $R=$ $8.27 \mathrm{kpc}$. However, in reality, any spiral arm in a disc galaxy has a finite radial extent. Here, we study how the group velocity of a wavepacket changes at different radii for different thickness, and 
Table 2. Radial variation in group velocity for a one-component stellar disc (with $Q=1.1, \Omega_{\mathrm{p}}=12.5 \mathrm{~km} \mathrm{~s}^{-1} \mathrm{kpc}^{-1}$ ), calculated at three radial locations.

\begin{tabular}{lccccc}
\hline \hline $\begin{array}{l}R \\
(\mathrm{kpc})\end{array}$ & $|s|_{\text {obs }}$ & $\beta$ & $\mathrm{d} s / \mathrm{d} x$ & $\begin{array}{c}v_{\mathrm{g}} \\
\left(\mathrm{km} \mathrm{s}^{-1}\right)\end{array}$ & $\begin{array}{c}\text { Time to travel } \\
10 \mathrm{kpc}(\mathrm{Gyr})\end{array}$ \\
\hline 6.27 & 0.94 & 0 & 0.021 & 1.14 & 8.5 \\
& & 0.1 & 0.027 & 1.46 & 6.7 \\
& & 0.2 & 0.032 & 1.71 & 5.7 \\
& & 0.25 & 0.034 & 1.82 & 5.35 \\
7.27 & 0.87 & 0.3 & 0.035 & 1.9 & 5.1 \\
\hline & & 0.1 & 0.07 & 2.8 & 3.5 \\
& & 0.2 & 0.083 & 3.42 & 2.85 \\
& & 0.25 & 0.086 & 3.9 & 2.56 \\
\hline 9.27 & 0.72 & 0.3 & 0.09 & 4.2 & 2.45 \\
& & 0.1 & 0.156 & 5.6 & 1.73 \\
& & 0.2 & 0.184 & 6.4 & 1.51 \\
& & 0.25 & 0.188 & 6.8 & 1.45 \\
& & 0.3 & 0.192 & 6.9 & 1.43 \\
\hline & & & & & 1.41 \\
\hline
\end{tabular}

consequently, how this affects the longevity of the spiral density wave.

We mention that for an assumed flat rotation curve in the outer disc region, as done here, the values of $\kappa$ and $\Omega$ change at different radial locations. Therefore, for an assumed value of $\Omega_{\mathrm{p}}$ that remains constant with respect to radius, the corresponding $|s|_{\text {obs }}$ value would change at different radial locations, and so would the $x$ values where the $|s|_{\text {obs }}$ cuts the local dispersion relation. To evaluate this, we first took $\Omega_{\mathrm{p}}=12.5 \mathrm{~km} \mathrm{~s}^{-1} \mathrm{kpc}^{-1}$ and $Q=1.1$ (same as Table 1, which was calculated for $R=8.27 \mathrm{kpc}$ ), and repeated the group velocity calculation at $R=6.27 \mathrm{kpc}, R=7.27 \mathrm{kpc}$, and $R=9.27 \mathrm{kpc}$. The results are given in Table 2 . This table clearly shows that at the three different radial locations we considered here, the variation in the finite thickness from $\beta=0$ to $\beta=0.3$ leads to a monotonic increase in the group velocity of the density wavepacket. To express this more quantitatively, at $R=6.27 \mathrm{kpc}$, the group velocity increases by $\sim 66.2 \%$ when $\beta$ is varied from 0 to 0.3 , whereas at $R=7.27 \mathrm{kpc}$ and $R=9.27 \mathrm{kpc}$, the corresponding group velocity increases by $\sim 49.8$ and $\sim 23.1 \%$, respectively (for the same $\beta$ variation). This trend agrees with that seen at $R=8.27 \mathrm{kpc}$ (see Table 1). In other words, the finite thickness has a similar (qualitative) effect on the group velocity and on the longevity of spiral density waves. We checked this trend for the other considered pattern values, namely, $\Omega_{\mathrm{p}}=15 \mathrm{~km} \mathrm{~s}^{-1} \mathrm{kpc}^{-1}$ and $\Omega_{\mathrm{p}}=10 \mathrm{~km} \mathrm{~s}^{-1} \mathrm{kpc}^{-1}$ as well. We found a qualitative trend in the results similar to what is seen for $\Omega_{\mathrm{p}}=12.5 \mathrm{~km} \mathrm{~s}^{-1} \mathrm{kpc}^{-1}$, as long as the dispersion relation admits a real solution in $x$ in the short-wavelength branch for the corresponding $|s|_{\text {obs }}$ value (for details, see the previous section). For brevity they are not shown here. To conclude, the finite thickness of the stellar disc has a similar quenching effect on the longevity of spiral density waves at different radial locations (covering the radial extent of spirals).

\subsection{Effect of finite height on the two-component star-gas system}

We showed above that including a finite thickness of the stellar disc makes it more stable against the non-axisymmetric per-
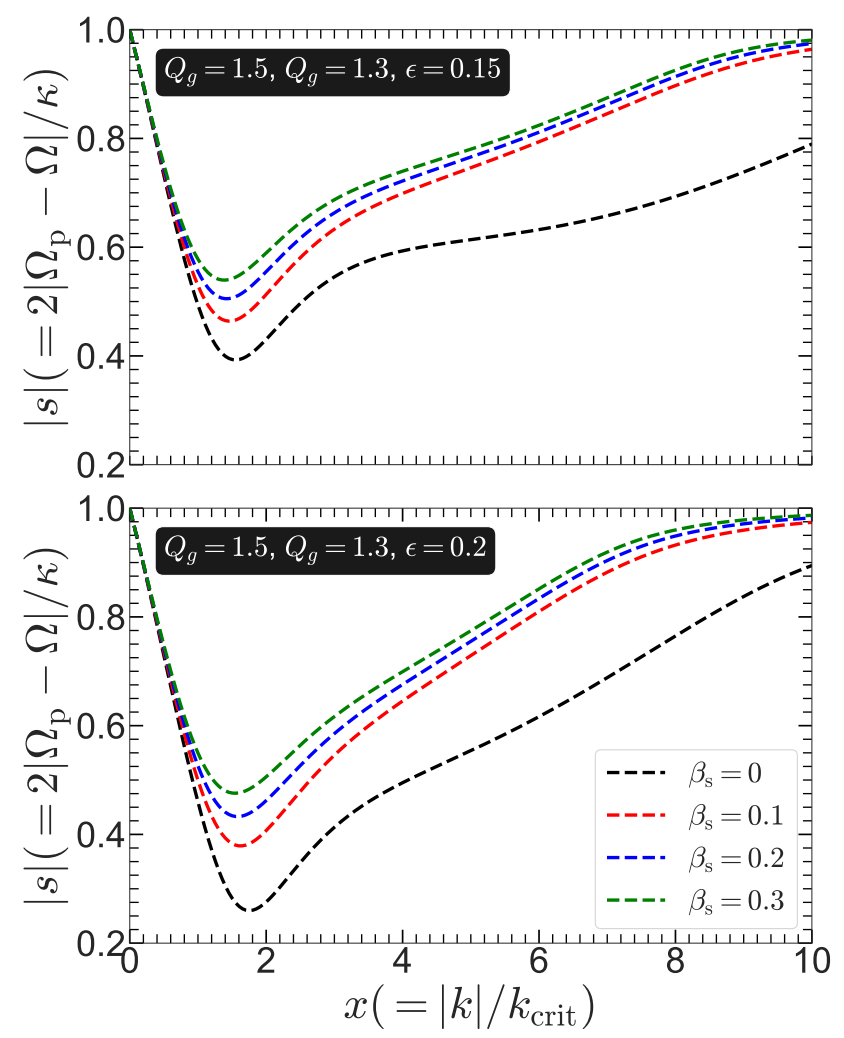

Fig. 3. Dispersion relations for the two-component stars plus gas system with finite thickness (Eq. (46)) are shown for $Q_{\mathrm{s}}=1.5, Q_{\mathrm{g}}=1.3$, and for different gas fractions: $\epsilon=0.15$ (top panel) and $\epsilon=0.2$ (bottom panel). The thickness of the stellar disc $\left(\beta_{\mathrm{s}}\right)$ is varied from 0.1 to 0.3 , whereas the thickness of the gas disc $\left(\beta_{\mathrm{g}}\right)$ is kept fixed at 0.1 throughout all cases shown here. For comparison, the corresponding dispersion relation for the zero-thickness stars plus gas system is also shown (dashed black line).

turbations and increases the group velocity of a wavepacket, thereby decreasing the longevity of the spiral density wave. Furthermore, Ghosh \& Jog (2015) showed that for a gravitationally coupled star-gas system in which the stellar and the gas discs are both infinitesimally thin, including the gas helps the spiral density waves to last for a longer time. Therefore, it is natural to investigate how the longevity of the spiral density wave is affected in a stars plus gas system with finite disc thickness.

To achieve this, first we calculated the dispersion relation (Eq. (46)) for the gravitationally coupled star-gas system for different thickness values of the stellar disc $\left(\beta_{\mathrm{s}}\right)$ while assuming $Q_{\mathrm{s}}=1.5, Q_{\mathrm{g}}=1.3$, and taking two values of $\epsilon$, namely, 0.15 and 0.2 . We mention that the thickness of the gas disc $\left(\beta_{\mathrm{g}}\right)$ was kept fixed at 0.1 throughout this paper, unless stated otherwise. The resulting dispersion relations are shown in Fig. 3. A visual inspection reveals two broad trends, namely, for a fixed set of three values $\left(Q_{\mathrm{s}}, Q_{\mathrm{g}}\right.$, and $\left.\epsilon\right)$, the value of $|s|_{\text {cut-off }}$ increases monotonically with increasing thickness of the stellar disc. This trend is consistent with the findings for the onecomponent stellar disc. However, we note that with increasing gas fraction $(\epsilon)$, the increment in the $|s|_{\text {cut-off }}$ value with thickness is smaller (compare the top and bottom panels of Fig. 3). Secondly, the dispersion relation in the short-wavelength branch becomes increasingly flat with increasing disc thickness when compared with the same for the infinitesimally thin star-gas system. This holds true for the two gas-fraction values considered here. 

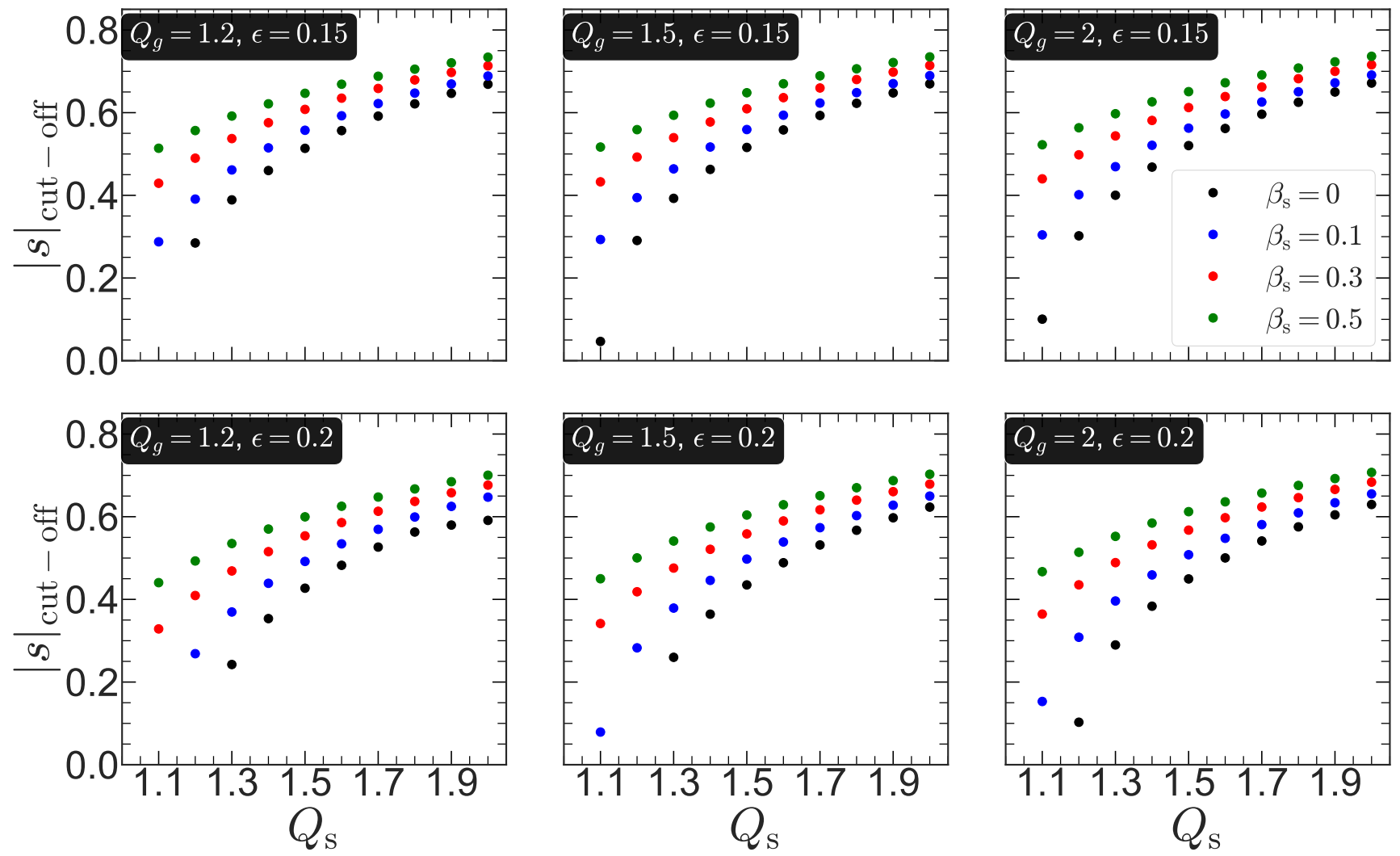

Fig. 4. Two-component stars plus gas system: variation in the $|s|_{\text {cut-off }}$ values as a function of Toomre $Q$ parameter for the stellar disc $\left(Q_{\mathrm{s}}\right)$ shown for different stellar disc thickness $\left(\beta_{\mathrm{s}}\right)$ and for various Toomre $Q$ parameter for the gas disc $\left(Q_{\mathrm{g}}\right)$ and gas-fraction $(\epsilon)$. The thickness of the gas disc $\left(\beta_{\mathrm{g}}\right)$ is kept fixed at 0.1 throughout all the cases shown here.

\subsubsection{Disc stability against spiral waves, and allowed pattern speed values}

In order to probe the joint effect of the disc thickness and the inclusion of gas on the variation of $|s|_{\text {cut-off }}$ values, we systematically calculated the relevant dispersion relations (using Eq. (46)) for a wide range of $Q_{\mathrm{s}}, Q_{\mathrm{g}}, \beta_{\mathrm{s}}$, and $\epsilon$. Then we computed the corresponding $|s|_{\text {cut-off }}$ values. The resulting variation in $|s|_{\text {cut-off }}$ values is shown in Fig. 4. Figure 4 clearly demonstrates the generic trend that for a fixed value of $\left(Q_{\mathrm{s}}, Q_{\mathrm{g}}, \epsilon\right)$, the $|s|_{\text {cut-off }}$ increases monotonically, while the disc thickness $\left(\beta_{\mathrm{s}}\right)$ is varied from 0 to 0.5 . However, the variation in the $|s|_{\text {cut-off }}$ values is stronger for a lower $Q_{\mathrm{s}}$ value (e.g. 1.2) when compared with a higher $Q_{\text {s }}$ value (e.g. $\left.\sim 2\right)$. These findings are in compliance with the earlier finding for the one-component stellar disc. Next, we study how the variation in the $|s|_{\text {cut-off }}$ values with thickness is affected when interstellar gas is included. We find that for a fixed value of $\left(Q_{\mathrm{g}}, \beta_{\mathrm{s}}\right)$, the $|s|_{\text {cut-off }}$ value for a higher gas fraction $(\epsilon)$ is always lower when compared with the same for a lower gas fraction (compare the top and bottom panels of Fig. 4). This trend holds true for the whole range of $Q_{\mathrm{s}}$ and $Q_{\mathrm{g}}$ values considered here, although the trend is more prominent for lower values of $Q_{\mathrm{s}}$. The physical reason behind this trend is that when more gas is present in the system (a lower $Q_{\mathrm{g}}$ value and a higher $\epsilon$ value), the destabilising effect of the interstellar gas dominates the stabilising effect of the finite thickness. This is particularly true when the $Q_{\mathrm{s}}$ and $Q_{\mathrm{g}}$ values are lower (e.g. close to 1 ) as the self-gravity of the stars-gas system would be important to determine the dynamical state of the system. Thus, the net stability of the gravitationally coupled stars plus gas system is determined by the joint (mutually opposite) effects of the interstellar gas and the finite disc thickness. Nevertheless, the destabilising effect of the interstellar gas is reduced (at least partially) by the stabilising effect of the finite disc thickness in all cases shown here. Thus, the net range of allowed pattern speed values is wider for a twocomponent, finite-height disc than for a stars-alone, thin disk; but is is smaller than the range allowed for a two-component thin disc.

\subsubsection{Radial group transport and effect on the longevity of spiral waves}

Finally, we probed the joint effects of the disc finite thickness and the interstellar gas on the group velocity of the spiral density wave in a gravitationally coupled stars-gas system. For the sake of theoretical interest, we chose a lower value of $Q_{\mathrm{s}}$, for instance, 1.3. For the moment, we also treat $Q_{\mathrm{s}}$ and $\epsilon$ as free parameters, and $Q_{\mathrm{g}}$ is set by these two values via the relation $Q_{\mathrm{g}}=\left(0.306 Q_{\mathrm{s}}\right)(1-\epsilon) / \epsilon$ (for details, see Ghosh \& Jog 2015). We relax this constraint later and treat all three parameters, namely, $Q_{\mathrm{s}}, Q_{\mathrm{g}}$, and $\epsilon$, as free parameters while studying the group velocity.

To study how the group velocity changes, we first assumed $\Omega_{\mathrm{p}}=12.5 \mathrm{~km} \mathrm{~s}^{-1} \mathrm{kpc}^{-1}$, which in turn gives $|s|_{\mathrm{obs}}=0.8$ at $R=8.27 \mathrm{kpc}$. Then we computed the group velocity from the slopes of the dispersion relations (Eq. (46)) for $Q_{\mathrm{s}}=1.3$ and $\beta_{\mathrm{g}}=0.1$ while varying the gas fraction $(\epsilon)$ from 0.1 to 0.25 and the disc thickness $\left(\beta_{\mathrm{s}}\right)$ from 0 to 0.3 . The resulting variations in the slopes and the group velocity values are listed in Table 3. As evident from Table 3, the value of the group velocity increases with increasing disc thickness, and this trend holds true for all 
Table 3. Group velocity for a two-component star-gas system (with $Q_{\mathrm{s}}=1.3$ ) for various thickness values, calculated at $R=8.27 \mathrm{kpc}$.

\begin{tabular}{lcccccc}
\hline $\begin{array}{l}\Omega_{\mathrm{p}} \\
\left(\mathrm{km} \mathrm{s}^{-1}\right. \\
\left.\mathrm{kpc}^{-1}\right)\end{array}$ & $|s|_{\text {obs }}$ & $\epsilon$ & $\beta$ & $\mathrm{d} s / \mathrm{d} x$ & $\begin{array}{c}v_{\mathrm{g}} \\
\left(\mathrm{km} \mathrm{s}^{-1}\right)\end{array}$ & $\begin{array}{c}\text { Time to travel } \\
10 \mathrm{kpc}(\mathrm{Gyr})\end{array}$ \\
\hline 12.5 & 0.8 & 0.1 & 0 & 0.081 & 3.3 & 2.95 \\
& & & 0.1 & 0.1 & 4.1 & 2.37 \\
& & & 0.2 & 0.104 & 4.2 & 2.32 \\
& & 0.3 & 0.11 & 4.5 & 2.16 \\
\hline & 0.15 & 0 & 0.067 & 2.72 & 3.58 \\
& & 0.1 & 0.082 & 3.35 & 2.9 \\
& & 0.2 & 0.083 & 3.4 & 2.86 \\
& & 0.3 & 0.084 & 3.41 & 2.85 \\
\hline & 0.2 & 0 & 0.074 & 3 & 3.24 \\
& & 0.1 & 0.083 & 3.37 & 2.89 \\
& & 0.2 & 0.0832 & 3.38 & 2.88 \\
& & 0.3 & 0.0833 & 3.39 & 2.87 \\
\hline & 0.25 & 0 & - & - & - \\
& & 0.1 & 0.093 & 3.4 & 2.86 \\
& & 0.2 & 0.095 & 3.42 & 2.84 \\
& & 0.3 & 0.095 & 3.42 & 2.84 \\
\hline
\end{tabular}

Notes. The Toomre $Q$ for the gas disc $\left(Q_{\mathrm{g}}\right)$ is set by the values of $Q_{\mathrm{s}}$ and $\epsilon$; for details, see text. The thickness of the gas disc $\beta_{\mathrm{g}}$ is fixed at 0.1 for all cases.

values of the gas fraction considered here. However, there is a subtle change with increasing gas fraction. To elaborate, when the gas fraction is assumed to be 0.1 , the group velocity increases by $\sim 35 \%$ for a variation of $0-0.3$ in the $\beta$ values. However, for a higher gas fraction value (e.g. $\epsilon=0.2$ ), the group velocity increases by only $\sim 12 \%$ for the same variation of $\beta$ values from 0 to 0.3 . Furthermore, when we chose an even higher value of the gas-fraction (e.g. $\epsilon=0.25$ ), we find a negligible increase in the group velocity $(\sim 2 \%)$ when we vary $\beta$ from 0 to 0.3 . The physical reason is that as we chose progressively lower values of $Q_{\mathrm{g}}$ and higher values of $\epsilon$, the overwhelming effect of the interstellar gas in decreasing the group velocity dominates the opposite effect of disc thickness on the group velocity values. In other words, when the self-gravity and the low dispersion of the interstellar gas dominates the reduction in the self-gravity due to the finite thickness, the group velocity of a wavepacket, which consists of spiral density waves, is predominantly set by the effect of the interstellar gas. Next, we chose a higher pattern speed value, namely, $\Omega_{\mathrm{p}}=15 \mathrm{~km} \mathrm{~s}^{-1} \mathrm{kpc}^{-1}$, which in turn produced $|s|_{\text {obs }}=0.68 R=8.27 \mathrm{kpc}$. Then, we studied how the group velocity changes when the gas fraction and the disc finite thickness were varied simultaneously. We find that when $\epsilon=0.1$, the group velocity increases by $\sim 20 \%$ when the disc thickness is increased from 0 to 0.3 . However, when the gas fraction is changed to a higher value (e.g. $\epsilon=0.2$ ), the group velocity increases only by $\sim 14 \%$ when $\beta_{\mathrm{s}}$ is varied from 0 to 0.3 . This trend is similar to what we found for the $\Omega_{\mathrm{p}}=12.5 \mathrm{~km} \mathrm{~s}^{-1} \mathrm{kpc}^{-1}$. Thus, the change in group velocity in presence of the disc thickness and the interstellar gas is a complex process, as these two physical factors have opposite effects on the group velocity. The net change in the group velocity is set by the relative dominance of the effects of the interstellar gas and the disc thickness.

To further study the net effect of the interstellar gas and the disc thickness where the parameters $Q_{\mathrm{s}}, Q_{\mathrm{g}}$, and $\epsilon$ are treated as free parameters (unlike the previous case), we chose a case where $Q_{\mathrm{s}}=1.3$ and $Q_{\mathrm{g}}=1.3$. For these assumed parameters and for $\epsilon=0.2$ and $\Omega_{\mathrm{p}}=15 \mathrm{~km} \mathrm{~s}^{-1} \mathrm{kpc}^{-1}$, the group velocity increases by $\sim 25 \%$ when $\beta_{\mathrm{s}}$ is varied from 0 to 0.3 . However, for a higher $\epsilon=0.25$, the change in the group velocity is negligible when $\beta_{\mathrm{s}}$ is varied from 0 to 0.3 . This trend further demonstrates the mutual interplay of the (opposite) effects induced by the interstellar gas and the disc finite thickness. Lastly, we assumed an even higher value of the pattern speed, namely, $\Omega_{\mathrm{p}}=18 \mathrm{~km} \mathrm{~s}^{-1} \mathrm{kpc}^{-1}$, which in turn yielded $|s|_{\mathrm{obs}}=0.53$. For the currently assumed parameter space $\left(Q_{\mathrm{s}}, Q_{\mathrm{g}}, \epsilon, \beta_{\mathrm{s}}\right)$, it is possible to obtain a real solution in $k$ (or alternatively, in $x$ ) for the $\Omega_{\mathrm{p}}=18 \mathrm{~km} \mathrm{~s}^{-1} \mathrm{kpc}^{-1}$. This allows us to probe the mutual effect of the gas and the thickness for a realistic value (or close to observationally reported values) of $\Omega_{\mathrm{p}}$. Here also, we find the same trend in the change of the group velocity value as we increase the value of $\beta_{\mathrm{s}}$. More quantitatively, when $\epsilon=0.2$, the group velocity increases by $\sim 11 \%$ as we vary $\beta_{\mathrm{s}}$ from 0 to 0.3 . However, the change in the group velocity is found to be negligible when $\epsilon$ is set to 0.25 while $\beta_{\mathrm{s}}$ is varied by the same amount as in the previous case. This further accentuates the complex mutual interplay of the opposite effects of the disc thickness and the interstellar gas on the resulting group velocity values.

Lastly, we explored the mutual effect of the disc thickness and the interstellar gas for some higher values of $Q_{\mathrm{s}}$ while varying the thickness of the stellar disc up to $\sim 400 \mathrm{pc}$ (equivalently, $\left.\beta_{\mathrm{S}} \sim 0.5\right)$. This is close to the observed values of the disc thickness in the external galaxies (e.g. see de Grijs \& Peletier 1997). To do this, we first chose $Q_{\mathrm{s}}=1.6, Q_{\mathrm{g}}=1.5$, and $\epsilon=0.2$, and then varied the disc thickness $\left(\beta_{\mathrm{s}}\right)$ from 0 to 0.5 . We find that for this chosen set of values for $\left(Q_{\mathrm{s}}, Q_{\mathrm{g}}\right.$, and $\left.\epsilon\right)$, and for an assumed $\Omega_{\mathrm{p}}$ of $15 \mathrm{~km} \mathrm{~s}^{-1} \mathrm{kpc}^{-1}$, the group velocity increases monotonically with increasing disc thickness. We estimate that for a variation in the disc thickness from 0 to 0.5 , the group velocity increases by $\sim 37 \%$. Furthermore, we chose another set of values for $\left(Q_{\mathrm{s}}, Q_{\mathrm{g}}\right.$, and $\left.\epsilon\right)$, namely, $Q_{\mathrm{s}}=2, Q_{\mathrm{g}}=2$, and $\epsilon=0.2$. This set of chosen values is typical for the outer regions of the discs of Magellanic-type irregular galaxies where the gas fractions are high (e.g. see Gallagher \& Hunter 1984; Jog 1992). We then chose $\Omega_{\mathrm{p}}=12.5 \mathrm{~km} \mathrm{~s}^{-1} \mathrm{kpc}^{-1}$ and varied the disc thickness from 0 to 0.5 . We find that for this case, the group velocity increases by $\sim 13 \%$ as the disc thickness is varied from 0 to 0.5 . For this case, we could not use a higher pattern speed value, for example, $\Omega_{\mathrm{p}}=15 \mathrm{~km} \mathrm{~s}^{-1} \mathrm{kpc}^{-1}$ or higher, as the group velocity approach cannot be applied here. The reason is that the $|s|_{\text {obs }}$ values corresponding to these higher pattern speed values are almost always lower than the $|s|_{\text {cut-off }}$ values obtained from the dispersion relations corresponding to $Q_{\mathrm{s}}=2, Q_{\mathrm{g}}=2$, and $\epsilon=0.2$, and $\beta_{\mathrm{s}}=0.2-0.5$.

\subsubsection{Coverage of the parameter space}

Thus, to conclude, we studied the joint effect of the disc thickness and the interstellar gas on the stability of the disc against non-axisymmetric perturbations as well as on the longevity of the $m=2$ spiral density waves. By exploring a range of parameter space, we demonstrate the complex nature of the mutually opposite effect of the disc thickness and the interstellar gas. We mention that, while we studied this mutually opposite effect on the longevity of the spiral density wave, we could not carry out a systematic search in the parameter space, unlike in the case of disc stability (or equivalently, the variation in $|s|_{\text {cut-off }}$ ). The reasons are that first, the group velocity was obtained using the slope of the local dispersion relation graphically (as in Toomre 1969). Although this approach allowed us to calculate the group velocity conveniently, this procedure is not robust (for details, see Toomre 1969; Ghosh \& Jog 2015). The slope depends critically 
on the exact location of $x$ where the $|s|_{\text {obs }}$ (corresponding to a $\Omega_{\mathrm{p}}$ value) intersects the dispersion relation. In other words, it is a local value. Secondly, for a two-component system, the dispersion relation is a fourth-order polynomial (e.g. Jog \& Solomon 1984). This means that for a certain choice of parameters, if the $|s|_{\text {obs }}$ intersects the dispersion relation in the region of high $x$ that falls in the region of second minimum, the slope would tend to flatten, regardless of whether the solution in $x$, corresponding to this $|s|_{\text {obs }}$ value, is real or imaginary. Hence, while normally, a higher $\beta$ or thickness leads to a steeper slope, the flattening effect due to the $k^{4}$ behaviour may dominate in these cases, and hence a smaller slope may be obtained for higher $\beta$, which in turn leads to a lower value of the group velocity. This is opposite to the typical dependence on disc thickness shown above, where a higher $\beta$ was shown to result in a higher group velocity. In these cases, the effect of disc thickness would not be opposite, but would show a similar trend to the effect of gas. This complex, mixed behaviour is more likely to be seen at high gas fraction (see Jog \& Solomon 1984) or high $|s|_{\text {obs. }}$. This could contribute to a diverse and complex dynamical behaviour. The results obtained from varying the $\beta$ parameter for a fixed set of $\left(Q_{\mathrm{s}}, Q_{\mathrm{g}}, \epsilon\right)$ values therefore have to be interpreted with caution.

Moreover, we kept the thickness of the gas disc, $\beta_{\mathrm{g}}=0.1$ so far while calculating the dispersion relations for a two-component stars-plus-gas system. Using the $k_{\text {crit }}$ value mentioned in Sect. 3.1, this corresponds to $h_{\mathrm{g}} \sim 70 \mathrm{pc}$, which is the typical scale height for molecular hydrogen gas (e.g. Scoville \& Sanders 1987). On the other hand, neutral hydrogen ( $\mathrm{H} \mathrm{I}$ ) shows a typical value for the scale height of $\sim 150 \mathrm{pc}$ (e.g. Lockman 1984), which corresponds to $\beta_{\mathrm{g}}=0.2$. Here, we briefly state what happens to the findings mentioned above when we set $\beta_{\mathrm{g}}=0.2$ instead of $\beta_{\mathrm{g}}=0.1$. We chose $Q_{\mathrm{s}}=1.3, Q_{\mathrm{g}}=1.3$, and $\epsilon=0.2$, which we explored before in Sect. 4.2. We then set $\beta_{\mathrm{g}}=0.2$ and varied $\beta_{\mathrm{s}}$ from 0 to 0.3 , as before, to determine the change in the radial group velocity corresponding to $\Omega_{\mathrm{p}}=15 \mathrm{~km} \mathrm{~s}^{-1} \mathrm{kpc}^{-1}$ and $\Omega_{\mathrm{p}}=18 \mathrm{~km} \mathrm{~s}^{-1} \mathrm{kpc}^{-1}$. For fixed $\beta_{\mathrm{s}}$ and $\Omega_{\mathrm{p}}$ values, the group velocity increased by $\sim 13-55 \%$ for $\beta_{\mathrm{g}}=0.2$ when compared with that for $\beta_{\mathrm{g}}=0.1$. This shows that the finite thickness of the gas disc also increases the group velocity for the joint disc (and consequently, decreases the longevity) in a similar fashion as the thickness of the stellar disc. We also considered a few other sets of $\left(Q_{\mathrm{s}}, Q_{\mathrm{g}}\right.$, and $\left.\epsilon\right)$ values to test this. We find a general trend of an increasing group velocity with increasing thickness of the gas disc. For brevity, we do not show these cases here.

Nevertheless, we stress that in general, the inclusion of thickness of the stellar disc and the interstellar gas has opposing effects on the disc stability against non-axisymmetric perturbations and the radial group transport, as illustrated in terms of the typical examples in this section (also see Table 3). In general, for the observed disc thickness and gas fraction values, as we have discussed in this section, the quenching effect of the height does not (completely) overcome the supporting role played by the gas, and hence a disc would still be expected to host non-axisymmetric features whose longevity will be supported by the gas.

\subsubsection{Radial variation of the group transport for a two-component disc}

As before, we study here the radial variation of the effect of a finite thickness on the group velocity of a wavepacket, consisting of density waves, for a gravitationally coupled stars-gas system. As before, we chose three radial locations at $R=6.27 \mathrm{kpc}$, $R=7.27 \mathrm{kpc}$, and $R=9.27 \mathrm{kpc}$. Then we assumed $\Omega_{\mathrm{p}}=$ $12.5 \mathrm{~km} \mathrm{~s}^{-1} \mathrm{kpc}^{-1}$ and recalculated the group velocities for dif- ferent disc thickness and the gas fraction values while choosing $Q_{\mathrm{s}}$ and $Q_{\mathrm{g}}$ values identical to those in Table 3. Here also, we find a similar qualitative trend of mutually opposing effects of the finite thickness and the interstellar gas on the group velocity as well as the longevity of the spiral density waves. To elaborate, at $R=6.27 \mathrm{kpc}$, and for $\epsilon=0.1$ and $\Omega_{\mathrm{p}}=12.5 \mathrm{~km} \mathrm{~s}^{-1} \mathrm{kpc}^{-1}$, the group velocity increases by $\sim 63.6 \%$ when $\beta_{\mathrm{s}}$ is varied from 0 to 0.3 . As the gas fraction $(\epsilon)$ increases, the increment in the group velocity due to a variation of $\beta$ from 0 to 0.3 starts to diminish monotonically. For $\epsilon=0.2$, the corresponding increment in the group velocity becomes $\sim 37.8 \%$, and for $\epsilon=0.25$, the increment in the group velocity becomes $\sim 33.7 \%$ (for the same variation in $\beta$ from 0 to 0.3 ). In other words, when the gas fraction increases, the dynamical effect of the interstellar gas in supporting the spiral density wave starts to dominate the quenching effect of the finite thickness. This trend is similar to what was shown for $R=8.27 \mathrm{kpc}$ (see Table 3 ). We further checked for other $\Omega_{\mathrm{p}}$ values as well as for another two radial locations considered here. We found that as long as the dispersion relation admits a real solution for $x$ in the long-wavelength branch for the corresponding $|s|_{\text {obs }}$ value, and as long as the $k^{4}$ behaviour does not interfere in the locations where the group velocity is being calculated (for details, see the discussion in previous section), we obtain a similar qualitative effect of the finite thickness on the group velocity and on the longevity of the spiral density wave. For brevity, they are not shown here. This shows that the finite thickness of the stellar disc has a similar quenching effect on the longevity of spiral density waves at different radial locations (covering the radial extent of spirals) for the gravitationally coupled two-component disc as well.

\section{Discussion}

Here, we discuss several relevant points.

At high values of the scale height, its effect begins to dominate the effect of gas. The net effect therefore is an increase in the group velocity, which would lead to a shorter lifetime of the density wave. A typical galaxy is known to show a flaring stellar disc, as was shown observationally by de Grijs \& Peletier (1997). This feature occurs naturally, as was shown by the theoretical modelling of a multicomponent galactic disc (Narayan \& Jog 2002a). The Milky Way stellar disc also shows flaring, as seen observationally from several surveys, for instance, 2MASS (Momany et al. 2006), SEGUE (López-Corredoira \& Molgó 2014), LAMOST (Wang et al. 2018), and LAMOST plus Gaia (Yu et al. 2021). This flaring was explained by theoretical modelling of a multicomponent disc (Narayan \& Jog 2002b), especially for the outer Galaxy by Sarkar \& Jog (2018). This could explain in a generic way why spiral features are rare in outer regions, even though these regions tend to be gas rich.

The formulation presented here employs the linear perturbation theory. However, the growth of the spirals in a real galaxy can enter the non-linear regime as well. The study by D'Onghia et al. (2013) demonstrated that the non-linear response of the disc to the non-axisymmetric perturbations can alter the lifetime of spiral instability in a disc galaxy. While the study presented here clearly demonstrated the mutually opposite effect of the interstellar gas and the disc thickness on the disc stability and persistence of spiral density wave, it will be worth pursuing this effect in numerical simulations of disc galaxies. We also mention that we modelled the spiral structure as a twodimensional structure for simplicity, as is typically done. However, some recent self-consistent simulations of the growth of 
spirals in disc galaxies (e.g. see Debattista 2014; Ghosh et al 2022) showed that spirals can well be vertically extended.

\section{Conclusion}

In summary, we investigated the dynamical impact of the disc thickness on the disc stability and on the longevity of the spiral density wave. To do this, we derived the dispersion relations in the WKB approximation for a collisionless stellar disc with finite thickness as well as for a gravitationally coupled stars-plus-gas system with different thickness for the stars and the gas discs. The main findings are listed below.

- The inclusion of finite thickness effectively reduces the selfgravity of the disc, which in turn makes the disc more stable against the non-axisymmetric perturbations. This result for a one-component stellar disc holds true for the whole range of Toomre- $Q$ parameters (1.2-2) and the disc thickness $(\sim 50-400 \mathrm{pc})$ considered in this work. This stabilising effect is more prominent for lower values of Toomre- $Q$, where the self-gravity of the disc is stronger.

- The stabilising effect of the finite disc thickness has consequences in setting up the allowed range of pattern speed values for which the system allows a non-evanescent spiral density wave. With increasing disc thickness, this allowed range of pattern speed values becomes progressively narrower, especially for lower values of Toomre- $Q$ parameter. For a joint stars-plus-gas system, the effect of gas is to increase the range of pattern speed values, while the thickness has an opposite effect. Typically, the net range of allowed pattern speed values is wider for a two-component, finite-height disc than for a stars-alone, infinitesimally thin disc.

- For a one-component stellar disc, the group velocity of a wavepacket increases monotonically with the increment of disc thickness, thereby implying a progressively shorter lifetime for the spiral density wave. For the same change in disc thickness by $\sim 250 \mathrm{pc}$, the reduction in lifetime can vary from $\sim 10-60 \%$ when compared to the case of an infinitesimally thin stellar disc. This reduction depends on the assumed pattern speed values and on the Toomre- $Q$ parameter. In a two-component system, as the gas fraction is increased, the increase in group velocity caused by the effect of finite height is reduced. Hence the inclusion of gas opposes the reduction in lifetime caused by the finite thickness.

- Even in the presence of the interstellar gas, the disc thickness tends to stabilise the two-component (stars-gas) system against the non-axisymmetric perturbation. This holds true for the whole parameter space $\left(Q_{\mathrm{s}}=1.2-2, Q_{\mathrm{g}}=1.2-2\right.$, and $\epsilon \sim 0.10-0.25)$ considered here. However, when the dynamical effect of gas is important (e.g. lower values of $Q_{\mathrm{g}}$, and/or higher $\epsilon$ values), the stabilising effect of the disc thickness becomes minimum.

- The disc thickness tends to diminish the lifetime of the spiral density waves, even when the gas is present. For the same change in disc thickness by $\sim 400 \mathrm{pc}$, the amount of reduction in the lifetime can vary from $\sim 5-40 \%$ when compared to an infinitesimally thin stars-plus-gas system, depending on the assumed pattern speed and the $Q_{\mathrm{s}}, Q_{\mathrm{g}}$, and gas fraction $(\epsilon)$ values. However, for gas-rich systems $(\epsilon \geq 0.25)$, the effect of a finite disc thickness is shown to be negligible.

Thus, to conclude, we demonstrated that the interstellar gas and the disc thickness have an opposite dynamical effect on the disc instability and on the persistence of spiral density waves. While the gas makes a galactic disc more susceptible to nonaxisymmetric perturbations and helps the spiral density wave to survive for a longer time, the disc thickness affects the system in the opposite way. Consequently, the net dynamical effect is set by the relative dominance of these two physical factors. For the broad range of parameter space we considered, the quenching effect of the height does not completely suppress the supporting role of the gas, and hence a disc would still be expected to host non-axisymmetric features whose longevity will be supported by the gas.

Acknowledgements. We thank the anonymous referee for useful comment which helped to improve this paper. C. J. thanks the DST, Government of India for support via a J. C. Bose fellowship (SB/S2/JCB-31/2014).

\section{References}

Athanassoula, E. 2012, MNRAS, 426, L46

Baba, J., Saitoh, T. R., \& Wada, K. 2013, ApJ, 763, 46

Binney, J., \& Tremaine, S. 2008, Galactic Dynamics: Second Edition (Princeton: Princeton University Press)

Bournaud, F., Combes, F., Jog, C. J., \& Puerari, I. 2005, A\&A, 438, 507

Burstein, D. 1979, ApJ, 234, 829

Buta, R. J., Sheth, K., Regan, M., et al. 2010, ApJS, 190, 147

Comerón, S., Elmegreen, B. G., Knapen, J. H., et al. 2011a, ApJ, 741, 28

Comerón, S., Knapen, J. H., Sheth, K., et al. 2011b, ApJ, 729, 18

Comerón, S., Salo, H., \& Knapen, J. H. 2018, A\&A, 610, A5

Debattista, V. P. 2014, MNRAS, 443, L1

de Grijs, R., \& Peletier, R. F. 1997, A\&A, 320, L21

Dobbs, C., \& Baba, J. 2014, PASA, 31, e035

Dobbs, C. L., Theis, C., Pringle, J. E., \& Bate, M. R. 2010, MNRAS, 403, 625

D'Onghia, E., Vogelsberger, M., \& Hernquist, L. 2013, ApJ, 766, 34

Elmegreen, D. M., \& Elmegreen, B. G. 2014, ApJ, 781, 11

Elmegreen, D. M., Elmegreen, B. G., Yau, A., et al. 2011, ApJ, 737, 32

Fathi, K., Toonen, S., Falcón-Barroso, J., et al. 2007, ApJ, 667, L137

Fathi, K., Beckman, J. E., Piñol-Ferrer, N., et al. 2009, ApJ, 704, 1657

Faure, C., Siebert, A., \& Famaey, B. 2014, MNRAS, 440, 2564

Gallagher, J. S., III, \& Hunter, D. A. 1984, ARA\&A, 22, 37

García de la Cruz, J., Martig, M., Minchev, I., \& James, P. 2021, MNRAS, 501 5105

Gerhard, O. 2002, in The Dynamics, Structure and History of Galaxies: A

Workshop in Honour of Professor Ken Freeman, eds. G. S. Da Costa, E. M.

Sadler, \& H. Jerjen, ASP Conf. Ser., 273, 73

Ghosh, S., \& Jog, C. J. 2015, MNRAS, 451, 1350

Ghosh, S., \& Jog, C. J. 2016, MNRAS, 459, 4057

Ghosh, S., \& Jog, C. J. 2018, A\&A, 617, A47

Ghosh, S., Saha, K., Jog, C. J., Combes, F., \& Di Matteo, P. 2021, ArXiv e-prints [arXiv:2105.05270]

Ghosh, S., Debattista, V. P., \& Khachaturyants, T. 2022, MNRAS, 511, 784

Gillessen, S., Eisenhauer, F., Trippe, S., et al. 2009, ApJ, 692, 1075

Goldreich, P., \& Lynden-Bell, D. 1965, MNRAS, 130, 125

Grand, R. J. J., Kawata, D., \& Cropper, M. 2012, MNRAS, 426, 167

Hodge, J. A., Smail, I., Walter, F., et al. 2019, ApJ, 876, 130

Jog, C. J. 1992, ApJ, 390, 378

Jog, C. J. 2014, AJ, 147, 132

Jog, C. J., \& Solomon, P. M. 1984, ApJ, 276, 114

Julian, W. H., \& Toomre, A. 1966, ApJ, 146, 810

Jurić, M., Ivezić, Ž., Brooks, A., et al. 2008, ApJ, 673, 864

Kruk, S. J., Lintott, C. J., Bamford, S. P., et al. 2018, MNRAS, 473, 4731

Li, C., Zhao, G., Jia, Y., et al. 2019, ApJ, 871, 208

Lighthill, M. J. 1965, J. Inst. Math. Appl., 1,

Lin, C. C., \& Shu, F. H. 1964, ApJ, 140, 646

Lin, C. C., \& Shu, F. H. 1966, Proc. Natl. Acad. Sci., 55, 229

Lockman, F. J. 1984, ApJ, 283, 90

López-Corredoira, M., \& Molgó, J. 2014, A\&A, 567, A106

Lynden-Bell, D., \& Kalnajs, A. J. 1972, MNRAS, 157, 1

Masset, F., \& Tagger, M. 1997, A\&A, 322, 442

McMillan, P. J., Kordopatis, G., Kunder, A., et al. 2018, MNRAS, 477, 5279

Mera, D., Chabrier, G., \& Schaeffer, R. 1998, A\&A, 330, 953

Momany, Y., Zaggia, S., Gilmore, G., et al. 2006, A\&A, 451, 515

Narayan, C. A., \& Jog, C. J. 2002a, A\&A, 390, L35

Narayan, C. A., \& Jog, C. J. 2002b, A\&A, 394, 89

Rix, H.-W., \& Zaritsky, D. 1995, ApJ, 447, 82

Roškar, R., Debattista, V. P., Quinn, T. R., Stinson, G. S., \& Wadsley, J. 2008 ,

ApJ, 684, L79

Saha, K., \& Elmegreen, B. 2016, ApJ, 826, L21

Salo, H., Laurikainen, E., Buta, R., \& Knapen, J. H. 2010, ApJ, 715, L56 
A\&A 658, A171 (2022)

Sarkar, S., \& Jog, C. J. 2018, A\&A, 617, A142

Sarkar, S., \& Jog, C. J. 2019, A\&A, 628, A58

Savchenko, S., Marchuk, A., Mosenkov, A., \& Grishunin, K. 2020, MNRAS, 493,390

Schönrich, R. 2012, MNRAS, 427, 274

Schönrich, R., \& Binney, J. 2009, MNRAS, 396, 203

Schönrich, R., Binney, J., \& Dehnen, W. 2010, MNRAS, 403, 1829

Schönrich, R., McMillan, P., \& Eyer, L. 2019, MNRAS, 487, 3568

Scoville, N. Z., \& Sanders, D. B. 1987, in $\mathrm{H}_{2}$ in the Galaxy, eds. D. J.

Hollenbach, \& H. A. Thronson, Jr., 134, 21

Sellwood, J. A. 2011, MNRAS, 410, 1637

Sellwood, J. A. 2012, ApJ, 751, 44

Sellwood, J. A., \& Binney, J. J. 2002, MNRAS, 336, 785

Sellwood, J. A., \& Carlberg, R. G. 1984, ApJ, 282, 61

Sellwood, J. A., \& Carlberg, R. G. 2019, MNRAS, 489, 116

Sellwood, J. A., \& Lin, D. N. C. 1989, MNRAS, 240, 991
Shu, F. H. 2016, ARA\&A, 54, 667

Toomre, A. 1964, ApJ, 139, 1217

Toomre, A. 1969, ApJ, 158, 899

Toomre, A. 1981, in Structure and Evolution of Normal Galaxies, eds. S. M. Fall, \& D. Lynden-Bell, 111

Toomre, A., \& Toomre, J. 1972, ApJ, 178, 623

Tsikoudi, V. 1979, ApJ, 234, 842

Wang, H.-F., Liu, C., Xu, Y., Wan, J.-C., \& Deng, L. 2018, MNRAS, 478, 3367

Weinberg, M. D. 1992, ApJ, 384, 81

Whitham, G. B. 1960, J. Fluid Mech., 9, 347

Willett, K. W., Galloway, M. A., Bamford, S. P., et al. 2017, MNRAS, 464, 4176

Yoachim, P., \& Dalcanton, J. J. 2006, AJ, 131, 226

Yu, S.-Y., Ho, L. C., Barth, A. J., \& Li, Z.-Y. 2018, ApJ, 862, 13

Yu, Z., Li, J., Chen, B., et al. 2021, ApJ, 912, 106

Zaritsky, D., Salo, H., Laurikainen, E., et al. 2013, ApJ, 772, 135 
S. Ghosh and C. J. Jog: Effect of disc thickness on the longevity of spiral density waves

\section{Appendix A: Behaviour of the reduction factor}

Fig. A.1 shows the variation in reduction factor, $\delta$ (Eq. 43) with dimensionless wavenumber $x$ for various disc thicknesses (or $\beta$ values). The reduction due to the disc thickness becomes severe for higher values of the disc thickness, as expected. The reduction factor is also seen to become progressively more important in the short-wavelength (large $x$ ) branch, as expected from the form of Eq. 43.

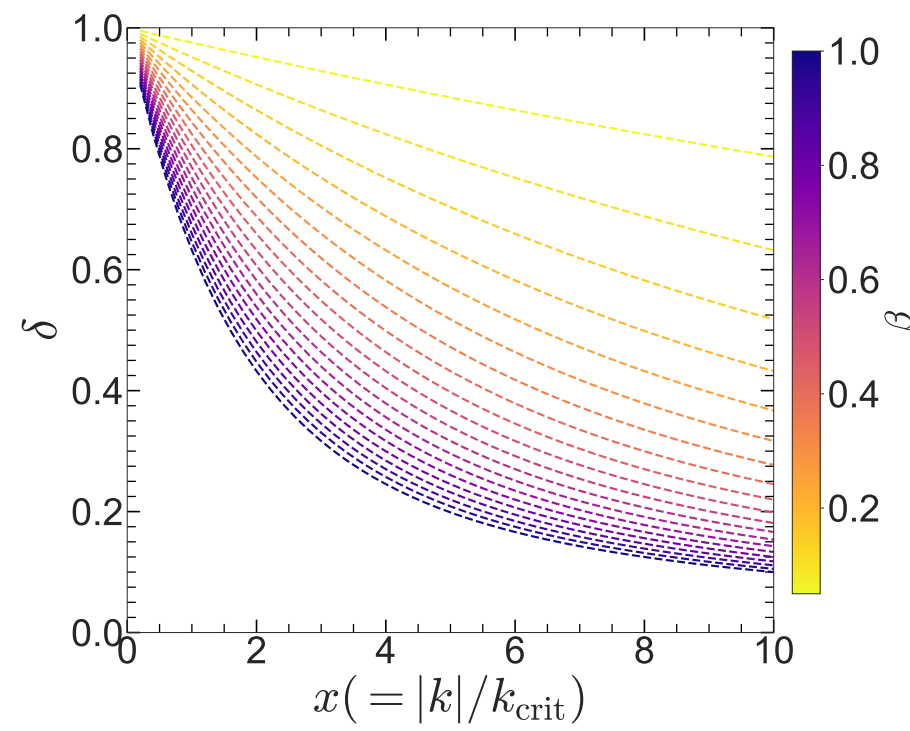

Fig. A.1. Variation in reduction factor $(\delta)$ as a function of dimensionless wavenumber $(x)$ shown for different disc thicknesses ( $\beta$ values). The values of $\beta$ are colour-coded here. 